CONFORMAL GEOMETRY AND DYNAMICS

An Electronic Journal of the American Mathematical Society

Volume 11, Pages 65-89 (June 5, 2007)

S $1088-4173(07) 00160-9$

\title{
PINCHING HOLOMORPHIC CORRESPONDENCES
}

\author{
SHAUN BULLETT AND PETER HAÏSSINSKY
}

\begin{abstract}
For certain classes of holomorphic correspondences which are matings between Kleinian groups and polynomials, we prove the existence of pinching deformations, analogous to Maskit's deformations of Kleinian groups which pinch loxodromic elements to parabolic elements. We apply our results to establish the existence of matings between quadratic maps and the modular group, for a large class of quadratic maps, and of matings between the quadratic map $z \rightarrow z^{2}$ and circle-packing representations of the free product $C_{2} * C_{3}$ of cyclic groups of order 2 and 3 .
\end{abstract}

\section{INTRODUCTION}

It is a well-known consequence of the simultaneous uniformisation theorem of Bers [2] that given two abstractly isomorphic Fuchsian groups $G_{1} \subset P S L_{2}(\mathbb{R})$ and $G_{2} \subset P S L_{2}(\mathbb{R})$, acting on the upper and lower complex half-planes respectively, each having limit set $\hat{\mathbb{R}}=\mathbb{R} \cup \infty$, and such that the action of $G_{1}$ on $\hat{\mathbb{R}}$ is topologically conjugate to that of $G_{2}$, the actions of $G_{1}$ and $G_{2}$ can be mated to obtain a quasifuchsian Kleinian group $G \subset P S L_{2}(\mathbb{C})$. This mating is a group which is abstractly isomorphic to both $G_{1}$ and $G_{2}$, it has limit set $\Lambda(G)$ a simple closed (fractal) curve, and the actions of $G$ on the two components of $\Omega=\hat{\mathbb{C}}-\Lambda$ are conformally conjugate to those of $G_{1}$ on $\mathcal{U}$ and $G_{2}$ on $\mathcal{L}$.

It is also well known that given two polynomial maps $P$ and $Q$ of the same degree $n$, in appropriate circumstances one can find a rational map $R$ which realises a mating between the actions of $P$ and $Q$ on their filled Julia sets, in a precise sense as defined for example in [13. A necessary condition for a mating between two quadratic polynomials $P: z \rightarrow z^{2}+c$ and $Q: z \rightarrow z^{2}+c^{\prime}$ to exist is that $c$ and $c^{\prime}$ should not belong to conjugate limbs of the connectivity locus (the Mandelbrot Set) in parameter space: this was first shown also to be a sufficient condition in the case that $P$ and $Q$ are postcritically finite [19, 20, and subsequently for much more general classes of $P$ and $Q$ [13].

In [5] the first examples of holomorphic correspondences realising matings between Fuchsian groups and polynomials were presented. Holomorphic correspondences on the Riemann sphere are multi-valued maps $f: z \rightarrow w$ defined by polynomial equations $p(z, w)=0$. Examples of holomorphic correspondences are those defined by a union of the graphs of some finite set of Möbius transformations, or by the graph of a rational map (or its inverse). We say that such a correspondence

Received by the editors June 19, 2006.

2000 Mathematics Subject Classification. Primary 37F05; Secondary 37F30.

Key words and phrases. Holomorphic correspondences, matings, quasiconformal deformations, pinching, circle-packing.

(C)2007 American Mathematical Society Reverts to public domain 28 years from publication 
has bidegree $(m: n)$ if a generic point $z$ has $n$ images $w$ and a generic point $w$ has $m$ inverse images $z$.

Definition 1.1. Let $q_{c}: z \rightarrow z^{2}+c$ be a quadratic map with connected filled Julia set $K\left(q_{c}\right)$. A holomorphic correspondence $f: z \rightarrow w$ of bidegree $(2: 2)$ is called a mating between $q_{c}$ and the modular group $P S L_{2}(\mathbb{Z})$ if:

(a) there exists a completely invariant open simply-connected region $\Omega \subset \hat{\mathbb{C}}$ and a conformal bijection $h$ from $\Omega$ to the upper half-plane conjugating the two branches of $\left.f\right|_{\Omega}$ to the pair of generators $z \rightarrow z+1, z \rightarrow z /(z+1)$ of $P S L_{2}(\mathbb{Z})$;

(b) the complement of $\Omega$ is the union of two closed sets $\Lambda_{-}$and $\Lambda_{+}$, which intersect in a single point and are equipped with homeomorphisms $h_{ \pm}: \Lambda_{ \pm} \rightarrow$ $K\left(q_{c}\right)$, conformal on interiors, respectively conjugating $f$ restricted to $\Lambda_{-}$as domain and codomain to $q_{c}$ on $K\left(q_{c}\right)$, and conjugating $f$ restricted to $\Lambda_{+}$as domain and codomain to $q_{c}^{-1}$ on $K\left(q_{c}\right)$.

In [5] the one parameter family of correspondences

$$
\left(\frac{a z+1}{z+1}\right)^{2}+\left(\frac{a z+1}{z+1}\right)\left(\frac{a w-1}{w-1}\right)+\left(\frac{a w-1}{w-1}\right)^{2}=3
$$

was shown to contain examples of matings between quadratic maps and the modular group. The following conjecture is implicit in the discussion in Sections 1 and 6 of that paper.

Conjecture 1.2. The family (1.1) of (2:2) correspondences contains matings between $P S L_{2}(\mathbb{Z})$ and every quadratic polynomial having a connected Julia set, that is to say every $z \rightarrow z^{2}+c$ with $c \in \mathcal{M}$, the Mandelbrot set.

Supporting evidence was provided by proofs for particular examples and numerical experiments suggesting the resemblance between the space of matings and the Mandelbrot set. However difficulties in adapting the theory of polynomial-like maps [9] to the setting of pinched polynomial-like maps prevented a proof.

A different question turned out to be easier to answer. The modular group may be considered as a representation of the free product $C_{2} * C_{3}$ of cyclic groups, of orders two and three, in $P S L_{2}(\mathbb{C})$. Up to conjugacy there is a one parameter family of such representations and in the parameter space there is a set $\mathcal{D}$, homeomorphic to a once-punctured closed disc, for which the representation is discrete and faithful. The modular group corresponds to a particular boundary point of $\mathcal{D}$. Let $r$ be any representation of $C_{2} * C_{3}$ corresponding to a parameter value in the interior $\mathcal{D}^{\circ}$ of $\mathcal{D}$. The ordinary set $\Omega(r)$ of the Kleinian group defined by such a representation $r$ is connected and the limit set $\Lambda(r)$ is a Cantor set. In 4 the notion of a mating between such a representation $r$ of $C_{2} * C_{3}$ and a quadratic polynomial $q_{c}: z \rightarrow z^{2}+c$ was introduced: $\Lambda_{-}$and $\Lambda_{+}$are now disjoint, and their complement $\Omega$ is canonically associated to $\Omega(r)$ (see Section 2.2). By the application of polynomial-like mapping theory, the following analogue of Conjecture 1.2 was proved in [4].

Theorem 1.3. For every quadratic map $q_{c}: z \rightarrow z^{2}+c$ with $c \in \mathcal{M}$ and every faithful discrete representation $r$ of $C_{2} * C_{3}$ in $P S L_{2}(\mathbb{C})$ having connected ordinary set, there exists a polynomial relation $p(z, w)=0$ defining a $(2: 2)$ correspondence which is a mating between $q_{c}$ and $r$.

An outline of the proof of Theorem 1.3 is presented in Section 2.2, as a prelude to applying pinching techniques to the matings it shows to exist. 
We describe an involution $J$ on $\hat{\mathbb{C}}$ as compatible with a mating $f$ if $(J \circ f) \cup I_{\widehat{\mathbb{C}}}$ is an equivalence relation, where $I_{\widehat{\mathbb{C}}}$ denotes the identity map on $\widehat{\mathbb{C}}$ and $(J \circ f) \cup I_{\widehat{\mathbb{C}}}$ denotes the $3: 3$ correspondence defined by the algebraic curve

$$
p(z, J(w))(z-w)=0 .
$$

(Here $p(z, w)=0$ is the curve defining $f$.)

Proposition 1.4. Every mating with a compatible involution is conjugate to a correspondence in the following two parameter family (also considered in [5]):

$$
\left(\frac{a z+1}{z+1}\right)^{2}+\left(\frac{a z+1}{z+1}\right)\left(\frac{a w-1}{w-1}\right)+\left(\frac{a w-1}{w-1}\right)^{2}=3 k .
$$

As we shall see, the matings constructed in [4 have compatible involutions, so they have representatives in the family (1.2), a fact observed in 4 but for which Proposition 1.4 (proved in Section 2) provides a more conceptual setting.

The basic idea of pinching can be seen in the process by which the modular group can be obtained from any chosen standard representation $r_{*}$ of $C_{2} * C_{3}$ lying in the interior of $\mathcal{D}$, that is to say a faithful discrete representation with connected ordinary set $\Omega\left(r_{*}\right)$ (and therefore limit set a Cantor set). We first recall that each Kleinian representation of $C_{2} * C_{3}$ comes equipped with a canonical involution $\chi$ which conjugates the generators $\sigma \in C_{2}$ and $\rho \in C_{3}$ to their inverses (see Section 2.1); we let $G$ denote the group $\langle\chi, \sigma, \rho\rangle$. For each rational number $p / q$ there is an $\operatorname{arc} \delta_{p / q}$ on the orbit space $\Sigma=\Omega\left(r_{*}\right) / G$ which lifts to simple closed geodesic $\tilde{\delta}_{p / q}$ of winding number $p / q$ on a certain torus $\tilde{\Sigma}$ double-covering $\Sigma$ (see Lemma 3.1 in Section 3.1 for details). The $\operatorname{arc} \delta_{p / q}$ lifts to an $\operatorname{arc} \alpha_{p / q}$ on $\Omega\left(r_{*}\right)$ together with its translates under $G$. This $\operatorname{arc} \alpha_{p / q}$ is precisely $\langle\langle g\rangle\rangle$-invariant for any loxodromic element $g \in G$ which stabilises it. (Here $\langle\langle g\rangle\rangle$ denotes the maximal elementary subgroup of $G$ containing $g$, and saying that an arc $\alpha$ is precisely $\langle\langle g\rangle\rangle$-invariant means that $\langle\langle g\rangle\rangle \alpha=\alpha$ and $h(\alpha) \cap \alpha=\emptyset$ for all $h \in G$ not in $\langle\langle g\rangle\rangle$.) In this situation Maskit's Theorem 15 states that the representation of $G$ in $P S L_{2}(\mathbb{C})$ can be deformed to one in which $\alpha_{p / q}$ and its translates under $G$ are pinched to points and $g$ becomes parabolic. We deduce that we may pinch $\delta_{0}$, and hence its lift $\alpha_{0}$, to a point, thereby deforming the representation $r_{*}$ of $C_{2} * C_{3}$ to the representation $P S L_{2}(\mathbb{Z})$, which lies on the boundary of the deformation space $\mathcal{D}$. Similarly for $p / q \neq 0$ we may pinch $\delta_{p / q}$ to a point and so deform the representation $r_{*}$ to a faithful discrete representation which we denote $r_{p / 2 q}$. This has ordinary set a disjoint union of a countable infinity of open round discs, and limit set a circle-packing. The representation $r_{p / 2 q}$ depends only on the value of $p / 2 q \bmod$ 2: pinching $\delta_{(2 n q+p) / q}$ in place of $\delta_{p / q}$ amounts to approaching the same limit representation $r_{p / 2 q}$ but by a non-isotopic path in $\mathcal{D}$. We remark that by a deep result of McMullen [17] the representations $r_{p / 2 q}$ are dense in the boundary of $\mathcal{D}$.

Recently, Haïssinsky [12, Cui [7, and Haïssinsky and Tan Lei [13] proved analogous results to Maskit's in the context of rational maps, showing that, under appropriate hypotheses, given a rational map $R$ and an $R$-invariant union of arcs joining attracting to repelling cycles, one can continuously deform the map in such a way that the arcs, and their pre-images, are pinched to points and the cycles become parabolic.

In this paper, we adapt the techniques of [12] and [13] to apply them to the holomorphic correspondences constructed in 4. In Section 3 for any correspondence 
$p_{0}(z, w)=0$ which is a mating between $r_{*}$ and $q_{c}$, and for any rational number $p / q$, we identify an arc $\gamma_{p / q}$ such that the grand orbit of $\gamma_{p / q}$ under the correspondence is a union of infinitely many disjoint copies of $\gamma_{p / q}$ (or of copies of a quotient of $\gamma_{p / q}$ by an involution), and such that pinching each connected component of this union to a point corresponds to deforming the representation $r_{*}$ to $r_{p / 2 q}$. We describe the pinching process formally as follows.

Definition 1.5. A convergent pinching deformation for $\gamma_{p / q}$ is a family of quasiconformal maps $\left(\varphi_{t}\right)_{0<t<1}$ of the Riemann sphere such that the conjugate correspondences $p_{t}$ defined by

$$
p_{t}(z, w)=p_{0}\left(\varphi_{t}^{-1}(z), \varphi_{t}^{-1}(w)\right)
$$

are holomorphic and satisfy the following:

- $\left(p_{t}, \varphi_{t}\right)$ are uniformly convergent to a pair $\left(p_{1}, \varphi_{1}\right)$ as $t$ tends to 1 ,

- the non-trivial fibres of $\varphi_{1}$ are exactly the closure of the connected components of the orbit of $\gamma_{p / q}$.

There are two technical conditions that we require the quadratic map $q_{c}$ to satisfy in order to apply the techniques of 13 to $\gamma_{0}$ :

(i) if the critical point 0 of $q_{c}$ is recurrent, the $\beta$-fixed point of $q_{c}$ is not in the $\omega$-limit set of 0 ;

(ii) $q_{c}$ is weakly hyperbolic; that is, there are constants $r>0$ and $\delta<\infty$ such that, for all $z \in J_{q} \backslash\{$ preparabolic points $\}$, there is a subsequence of iterates $\left(q^{n_{k}}\right)_{k}$ such that

$$
\operatorname{deg}\left(W_{k}(z) \stackrel{q^{n_{k}}}{\longrightarrow} D\left(q^{n_{k}}(z), r\right)\right) \leq \delta
$$

where $W_{k}(z)$ is the connected component of $q^{-n_{k}}\left(D\left(q^{n_{k}}(z), r\right)\right)$ containing $z$.

In Section 4 we prove:

Theorem 1.6. Let $p_{0}(z, w)$ be a mating between the representation $r_{*}$ and $q_{c}$, where $q_{c}$ satisfies conditions (i) and (ii) above. Then there exists a pinching deformation of $p_{0}$ such that $\left(p_{t}\right)_{0 \leq t<1}$ converges uniformly to a mating $p_{1}$ between $P S L_{2}(\mathbb{Z})$ and $q_{c}$.

Corollary 1.7. Conjecture 1.2 is true for all quadratic maps $q_{c}$ which satisfy conditions (i) and (ii).

The class of weakly hyperbolic quadratic maps is quite large: for example, it contains all quadratic maps which satisfy the Collet-Eckmann condition [18, and those which contain parabolic points.

We next investigate pinching $\gamma_{p / q}$, for $p / q \neq 0$. In Section 3.2 , we define the notion of a mating between the circle-packing representation $r_{p / 2 q}$ of $C_{2} * C_{3}$ and $q_{c}$. This generalises our earlier definition of a mating between $P S L_{2}(\mathbb{Z})$ and $q_{c}$, replacing $K\left(q_{c}\right)$ by a certain identification space $K\left(q_{c}\right) / \sim_{p / q}$ and replacing the condition that $\Lambda_{+} \cap \Lambda_{-}$be a point by the condition that it consists of $q$ points (the $p / q$ Sturmian orbit on the boundary of $\left.K\left(q_{c}\right)\right)$. We show that a mating between $r_{p / 2 q}$ and $q_{c}$ depends only on $p / q \bmod 1$. To avoid technical difficulties, we restrict attention to the special case that the quadratic map is $q_{0}: z \rightarrow z^{2}$. Using the techniques of [12], we prove the following:

Theorem 1.8. Let $p_{0}(z, w)$ be a mating between the representation $r_{*}$ and $q_{0}$, and let $p / q$ be any rational. Then there exists a pinching deformation of $p_{0}$ such that 

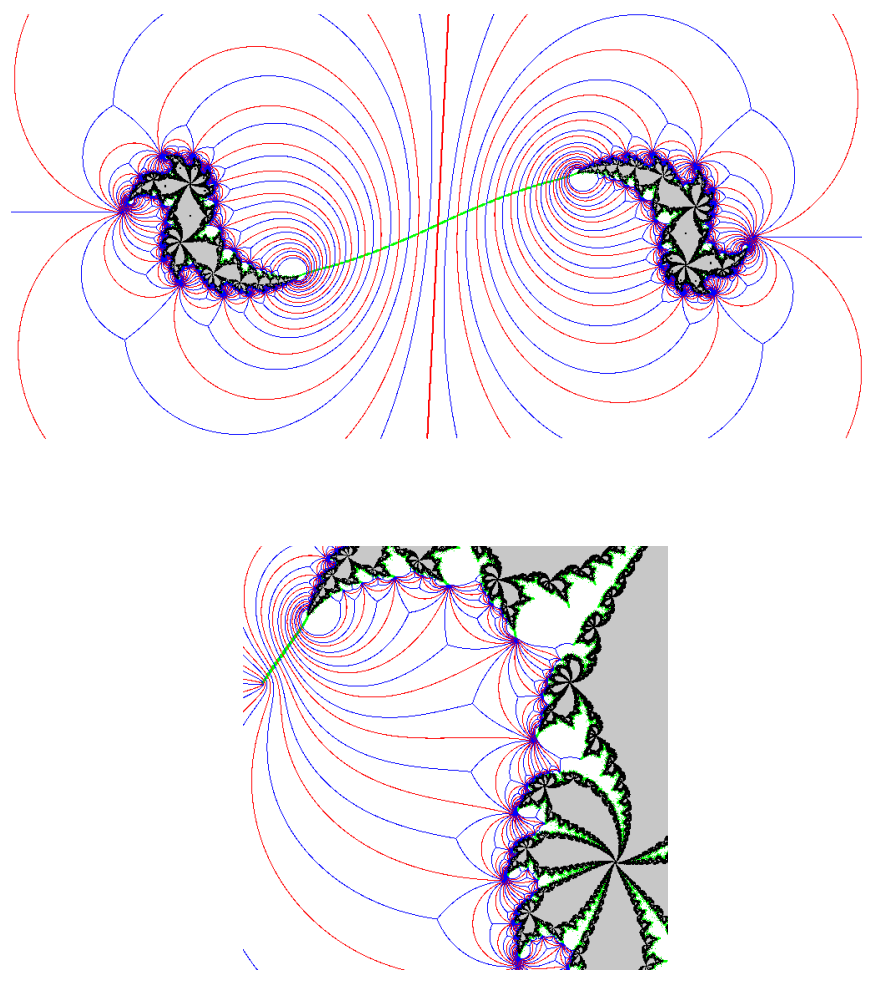

Figure 1. A mating of a representation of $C_{2} * C_{3}$ with a Douady rabbit (and zoom). The arc $\gamma_{0}$ and its images are shown. Pinching these gives a mating of $P S L_{2}(\mathbb{Z})$ with the rabbit, by Theorem 1.6 ,

$\left(p_{t}\right)_{0 \leq t<1}$ converges uniformly to a mating $p_{1}$ between the circle-packing representation $r_{p / 2 q}$ of $C_{2} * C_{3}$ in $P S L_{2}(\mathbb{C})$ and $q_{0}$.

The following is the natural generalisation of Conjecture 1.2 .

Conjecture 1.9. For every $0 \leq p / q<1$, the family (1.2) of (2:2) correspondences contains matings between the circle-packing representation $r_{p / 2 q}$ and every quadratic polynomial $q_{c}$ which has $c \in \mathcal{M} \backslash \mathcal{M}_{1-p / q}$, where $\mathcal{M}_{1-p / q}$ denotes the $(1-p / q)$-limb of the Mandelbrot set $\mathcal{M}$.

The condition that $c$ does not lie in $\mathcal{M}_{1-p / q}$ is necessary for elementary topological reasons. One might hope to generalise the techniques of the present paper to prove Conjecture 1.9 in the case that $q_{c}$ satisfies conditions (i) and (ii) of the hypotheses of Theorem 1.6. but the technical details could be formidable.

Warning. As will already be apparent, certain of the constructions and results in this article depend on $p / q \in \mathbb{Q}$, certain depend only on $p / q \bmod 1$ (the class of $p / q$ in $\mathbb{Q} / \mathbb{Z}$ ), and certain on $p / q$ mod 2 . We shall try to make the dependence clear in each case, but briefly the situation may be summed up as follows. A circle-packing representation $r_{p / 2 q}$ of $C_{2} * C_{3}$ depends on $p / q \bmod 2$ but the route to it (in the moduli space $\mathcal{D}$ ) given by pinching $\delta_{p / q}$ depends on $p / q \in \mathbb{Q}$. A mating between $r_{p / 2 q}$ of $C_{2} * C_{3}$ and $q_{c}$ depends only on $p / q \bmod 1$, but again the route to it (in mating space) given by pinching $\gamma_{p / q}$ depends on $p / q \in \mathbb{Q}$. 


\section{Matings Between quadratic maps And Representations of $C_{2} * C_{3}$}

We define what we mean by matings between quadratic maps and representations of $C_{2} * C_{3}$ in $P S L_{2}(\mathbb{C})$ which lie in $\mathcal{D}^{o}$, we recall the main ideas of the proof [4] of Theorem 1.3. we prove Proposition 1.4, and we present a group-theoretic description of the 'ordinary set' $\Omega(f)$ of a mating.

2.1. Faithful discrete representations with connected ordinary sets. Up to conjugacy, each representation $r$ of $C_{2} * C_{3}$ in $P S L_{2}(\mathbb{C})$ is determined by a single complex parameter, the cross-ratio between the fixed points on $\hat{\mathbb{C}}$ of the action of the generator $\sigma$ of $C_{2}$ and those of the generator $\rho$ of $C_{3}$. Such a representation comes equipped with a (unique) involution $\chi$ which exchanges the two fixed points of $\sigma$ and also those of $\rho$, so that $\chi \sigma=\sigma \chi$ and $\chi \rho=\rho^{-1} \chi$. On the Poincaré 3-ball, $\chi$ is simply rotation through $\pi$ around the common perpendicular to the axes of $\sigma$ and $\rho$. Write $G$ for the group $\langle\sigma, \rho, \chi\rangle$, and note that it has ordinary set $\Omega(G)$ the same as that of $\langle\sigma, \rho\rangle$.

The faithful discrete actions $r: C_{2} * C_{3} \subset P S L_{2}(\mathbb{C})$ with connected ordinary set $\Omega(G)$ form a single quasiconformal conjugacy class, the class of representations for which one can find simply-connected fundamental domains for $\sigma$ and $\rho$ with interiors together covering the whole Riemann sphere (the conditions of the simplest form of the Klein Combination Theorem are satisfied) [14]. Such fundamental domains may be constructed as illustrated in Figure 2

Here $P$ and $P^{\prime}$ are the fixed points of $\rho, Q$ and $Q^{\prime}$ are the fixed points of $\sigma, R$ is a fixed point of (the involution) $\chi \rho$ and $S$ and $S^{\prime}$ are the fixed points of $\chi \sigma$. The lines $l, m$ and $n$, joining $R$ to $S, Q$ to $S$ and $R$ to $P$, are chosen such that they are smooth and remain non-intersecting in the quotient orbifold $\Omega(G) / G$. The region bounded by $n, \rho n, \chi n$ and $\chi \rho n$ is a fundamental domain for $\rho$, and the region exterior to the loop made up of $m, \sigma m, \chi m$ and $\chi \sigma m$ is a fundamental domain for $\sigma$. The intersection of these two regions is a fundamental domain for the (faithful) action of $C_{2} * C_{3}$ on $\Omega(G)$, and the half $D_{G}$ of this intersection bounded by $n, l, m, \sigma m, \chi l$ and $\rho n$ is a fundamental domain for the action of $G$. The union of all translates of $D_{G}$ under elements of $C_{2} * C_{3}$ is a topological disc $D$ which is a fundamental domain for the action of $\chi$ on $\Omega(G)$. The complement $\Lambda(G)$ of $\Omega(G)=D \cup \chi(D)$ in $\hat{\mathbb{C}}$ is a Cantor set.

The orbifold $\Omega(G) / G$ is a sphere $\Sigma$, which has a complex structure with four cone points, which we may also label $P, Q, R, S$, where $P$ has angle $2 \pi / 3$ and $Q, R, S$ each have angle $\pi$. For a given representation of $C_{2} * C_{3}$, a set of lines $l, m, n$ as in Figure 2 descend to an isotopy class of non-intersecting paths joining the corresponding cone points in $\Sigma$. By considering the choices we may make of the various labels and lines in Figure 2, we can obtain a description of $\widetilde{\mathcal{D}}^{0}$, the universal cover of the moduli space $\mathcal{D}^{0}$.

Lemma 2.1. There is a homeomorphism $\Phi$ between $\mathcal{D}^{\circ}$ and the space $\mathcal{S}$ of spheres $\Sigma$ having a complex structure with four marked cone points $P, Q, R, S$ where $P$ has angle $2 \pi / 3$ and $Q, R, S$ each have angle $\pi$. This homeomorphism $\Phi$ lifts to a homeomorphism $\tilde{\Phi}$ between $\widetilde{\mathcal{D}}^{o}$ and the space $\tilde{\mathcal{S}}$ of spheres $\Sigma \in \mathcal{S}$ marked with an isotopy class of non-intersecting paths $P R, R S$ and $S Q$.

Proof. For $r \in \mathcal{D}^{o}$ define $\Phi(r)$ to be the orbifold $\Omega(G) / G$, where $G=\langle\sigma, \rho, \chi\rangle$ is the subgroup of $P S L_{2}(\mathbb{C})$ corresponding to the representation $r$. Clearly $\Phi$ is 


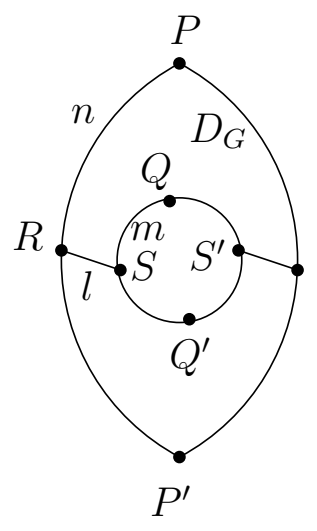

Figure 2. A fundamental domain $D_{G}$ for the group $G=\langle\sigma, \rho, \chi\rangle$.

continuous as $\mathcal{D}^{o}$ is endowed with the topology induced by its parametrisation by the cross-ratio $\left(Q, Q^{\prime} ; P, P^{\prime}\right)$. To define an inverse to $\Phi$, observe that given any $\Sigma \in \mathcal{S}$, we may obtain a representation $r$ by regarding $\Sigma$ as a quasiconformal deformation of the orbifold corresponding to $r_{*}$, lifting the corresponding ellipse field to $\hat{\mathbb{C}}$, and applying the Measurable Riemann Mapping Theorem.

To lift $\Phi$ to a homeomorphism $\tilde{\Phi}$ we have to consider markings. Note that given a representation of $C_{2} * C_{3}$ which lies in $\mathcal{D}^{o}$, there is only one choice for which of the pair $P, P^{\prime}$ (in Figure 2) to label $P$, namely the fixed point of $\rho$ around which the rotation is anti-clockwise. There is also just one choice (up to isotopy) for the arc $n$. The labels $Q$ and $Q^{\prime}$ are interchangeable (provided that we also interchange the labels $S$ and $S^{\prime}$ ), but once a choice has been made for $Q$ the arc $m$ is determined, and even if the labels $Q$ and $Q^{\prime}$ are exchanged, the arc $Q S$ in the orbifold $\Sigma$ is unchanged up to isotopy. This just leaves us a choice of the arc $l$ in Figure 2, We can alter $l$ to wind an extra $n$ times around the central 'hole' for any integer $n$, or $n+1 / 2$ times if we switch the labels $Q$ and $Q^{\prime}$. Changing the winding number of $l$ corresponds to choosing a different isotopy class of paths between the points labelled $R$ and $S$ in the orbifold $\Sigma$.

Let $t_{\alpha}$ denote the automorphism of $\widetilde{\mathcal{D}}^{o}$ corresponding to turning the internal boundary of Figure 2 through an angle $2 \pi \alpha$. Note that $t_{1 / 4}$ moves the pair of points labelled $Q, Q^{\prime}$ to the pair labelled $S, S^{\prime}$ and vice versa. Let $\iota: \mathcal{D}^{o} \rightarrow \mathcal{D}^{o}$ denote the involution obtained by replacing the generating pair $\{\sigma, \rho\}$ of $C_{2} * C_{3}$ by $\left\{\sigma^{\prime}, \rho\right\}$, where $\sigma^{\prime}=\chi \sigma$. This corresponds to composing the representation with an outer automorphism of $C_{2} * C_{3}$. The following result is now self-evident.

Lemma 2.2. The automorphism $t_{1 / 4}: \widetilde{\mathcal{D}}^{o} \rightarrow \widetilde{\mathcal{D}}^{o}$ covers $\iota: \mathcal{D}^{o} \rightarrow \mathcal{D}^{o}$, and $t_{1 / 2}$ generates the group of covering transformations of $\widetilde{\mathcal{D}}^{o} \rightarrow \mathcal{D}^{o}$.

2.2. Matings between $q_{c}$ and $r \in \mathcal{D}^{o}$. As in the previous subsection, $G$ denotes the group $\langle\sigma, \rho, \chi\rangle$.

Definition 2.3. A $(2: 2)$ holomorphic correspondence $f: z \rightarrow w$ is called a mating between a faithful discrete representation $r$ of $C_{2} * C_{3}$ in $P S L_{2}(\mathbb{C})$ having connected ordinary set $\Omega(G)$ and a polynomial $q_{c}: z \rightarrow z^{2}+c$ having connected filled Julia set $K\left(q_{c}\right)$, if the Riemann sphere $\hat{\mathbb{C}}$ is the disjoint union of a connected open set 

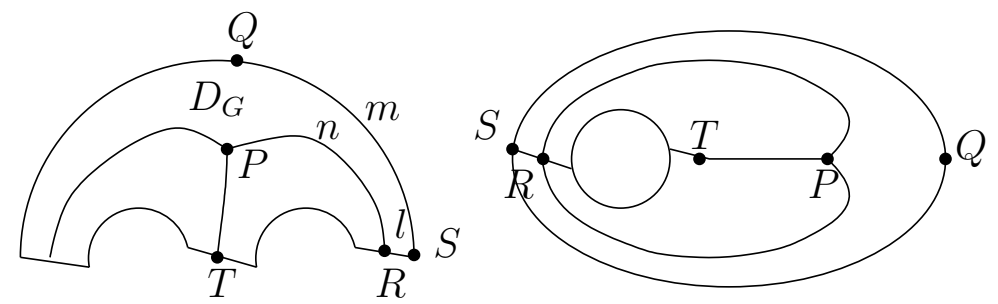

Figure 3. The set $D_{G} \cup \rho\left(D_{G}\right) \cup \rho^{-1}\left(D_{G}\right)$ and its quotient the annulus $B$.

$\Omega(f)$ and a closed set $\Lambda(f)$ made up of two components, $\Lambda_{+}(f)$ and $\Lambda_{-}(f)$ such that each of $\Omega(f)$ and $\Lambda(f)$ is completely invariant under $f$ and:

(a) the action of $f$ on $\Omega(f)$ is discontinuous and there is a conformal bijection between the grand orbit space $\Omega(f) / f$ and $\Omega(G) / G$;

(b) there is a quasiconformal homeomorphism defined from a neighbourhood of $\Lambda_{-}(f)$ onto a neighbourhood of $K\left(q_{c}\right)$ in $\mathbb{C}$, which realises a hybrid equivalence, conjugating $f$ to $q_{c}$. Similarly, there is a hybrid equivalence between $\left(f^{-1}, \Lambda_{+}(f)\right)$ and $\left(q_{c}, K\left(q_{c}\right)\right)$, this time conjugating $f^{-1}$ to $q_{c}$.

(See 9 for the definition of the term 'hybrid equivalence'.)

The construction of a holomorphic correspondence which realises a mating between given $q_{c}$ and $r$ proceeds as follows (see 44 for more details).

We first associate an annulus $A$ to $q_{c}: z \rightarrow z^{2}+c$. There is a holomorphic conjugacy (the Böttcher coordinate) from $z \rightarrow z^{2}$ to $q_{c}$ on a neighbourhood of $\infty$, fixing the point $\infty$ and tangent to the identity map there 8. An equipotential for $q_{c}$ is the image of a circle $\left\{R e^{2 \pi i t}: 0 \leq t<1\right\}$ under this conjugacy. It is a smooth Jordan curve parameterized by external angle $t$. The region bounded by such an equipotential is a simply-connected domain $V$, mapped $2: 1$ by $q_{c}$ onto a larger domain $U \supset \bar{V}$ which also has boundary an equipotential parametrised by external angle. Let $A$ denote the annulus $U-V$, and denote its inner and outer boundaries by $\partial_{1} A$ and $\partial_{2} A$, respectively. The map $q_{c}$ sends $\partial_{1} A$ two-to-one onto $\partial_{2} A$. There is an involution $i: z \rightarrow-z$ on $V$ sending each $z \in V$ to the other point which has the same image in $U$ under $q_{c}$, and there are many choices possible of an orientation-reversing smooth involution $j$ on $\partial_{2} A$, a canonical choice being given by $t \rightarrow 1-t$ on external angles.

The next ingredient is an annulus $B$ associated to $r$. Recall the fundamental domain $D_{G}$ constructed above for the group $G=\langle\sigma, \rho, \chi\rangle$ and illustrated in Figure 2. Let $B$ denote the annulus consisting of the three copies $D_{G} \cup \rho D_{G} \cup \rho^{-1} D_{G}$ of $D_{G}$, with the boundary identifications (induced by $\chi$ ) indicated in Figure 3 . The rotations $\rho$ and $\rho^{-1}$ mapping $D_{G} \cup \rho D_{G} \cup \rho^{-1} D_{G}$ to itself descend to a $2: 2$ correspondence $g$ on $B$, mapping each $z \in B$ to the pair $\left\{\rho z, \rho^{-1} z\right\}$ (or rather to their equivalence classes under the action of $\chi$ ). The set $D_{G}$ descends to a 'fundamental domain' for the action of $g$ on $B$. The boundary of $B$ is divided into three segments (two inner and one outer, Figure 3), each of which is mapped to the other two by $g$. Thus when its domain is restricted to the inner boundary $\partial_{1} B$, and its range is restricted to the outer boundary $\partial_{2} B$, the correspondence $g$ defines a two-to-one map. When restricted to a correspondence from the inner boundary to itself, $g$ defines a (fixed point free) bijection. Moreover, the involution $\sigma$ descends to an 
involution (which we also denote $\sigma$ ) on the outer boundary $\partial_{2} B$ of $B$, having fixed points $Q$ and $S$.

Lemma 2.4. There exists a quasiconformal homeomorphism $h$ from $A$ to $B$ which restricts to a smooth homeomorphism from $\partial A$ to $\partial B$ conjugating the boundary maps $\left(q_{c}: \partial_{1} A \rightarrow \partial_{2} A, j: \partial_{2} A \rightarrow \partial_{2} A\right)$ to the boundary maps $\left(\sigma \circ g: \partial_{1} B \rightarrow\right.$ $\left.\partial_{2} B, \sigma: \partial_{2} B \rightarrow \partial_{2} B\right)$.

This lemma is proved 4 by applying standard techniques of Ahlfors and Bers. Now to construct a mating between $q_{c}$ and $r$, first glue together $U$ and a second copy $U^{\prime}$ of $U$, via the boundary involution $j$, to obtain a sphere $U \cup U^{\prime}$, equipped with an involution (also denoted $j$ ) exchanging $U$ with $U^{\prime}$ and restricting to the original $j$ on the common boundary. Inside $U^{\prime}$ is a simply-connected subdomain $V^{\prime}$ corresponding to $V \subset U$. Let $q_{c}^{\prime}=j \circ q_{c} \circ j: V^{\prime} \rightarrow U^{\prime}$ denote the quadratic map corresponding to $q_{c}: V \rightarrow U$ and $A^{\prime}$ denote the annulus $U^{\prime}-V^{\prime}$. To define a $2: 2$ topological correspondence $f$ on $U \cup U^{\prime}$ we fit together:

- $q_{c}: V \rightarrow U$ (a $2: 1$ correspondence);

- $\left(q_{c}^{\prime}\right)^{-1}=j \circ q_{c}^{-1} \circ j: U^{\prime} \rightarrow V^{\prime}$ (a $1: 2$ correspondence);

- $j \circ i: V \rightarrow V^{\prime}$ (a $1: 1$ correspondence), and

- $j \circ g: A \rightarrow A^{\prime}$ (a $2: 2$ correspondence),

where $g: A \rightarrow A$ is the $2: 2$ correspondence constructed earlier. Now define an ellipse field on $A$ by using Lemma 2.4 to transport the standard complex structure from the annulus $B$. Using $j$, extend this ellipse field to $A^{\prime}$, and pulling back via $q_{c}^{-1}$ and $q_{c}^{\prime-1}$, extend it to an ellipse field on the whole of $\hat{\mathbb{C}}-\left(K\left(q_{c}\right) \cup K\left(q_{c}^{\prime}\right)\right)$, which transforms equivariantly under the action of the $2: 2$ correspondence $f$. Extend this ellipse field to the whole of $\hat{\mathbb{C}}$ by using the standard complex structure on $K\left(q_{c}\right) \cup K\left(q_{c}^{\prime}\right)$. By applying the Measurable Riemann Mapping Theorem we obtain a complex structure respected by $f$, completing our outline proof of Theorem 1.3

For any mating $f$ constructed by the method of the proof above, the $3: 3$ correspondence $(j \circ f) \cup I_{\widehat{\mathbb{C}}}$ sends each $z \in V$ to the triple of points $\left\{z, i(z), j q_{c}(z)\right\}$, each $z \in A$ to the triple $\{z, g(z)\}$ (recall that $g$ is $2: 2$ so $g(z)$ contains two points), and each $z \in U^{\prime}$ to the triple $\left\{z, q_{c}^{-1} j(z)\right\}$. It is easily checked that each of these triples is the grand orbit under $(j \circ f) \cup I_{\widehat{\mathbb{C}}}$ of any one of its elements, in other words the $3: 3$ correspondence is an equivalence relation. The involution $j$ is therefore compatible with the mating $f$ in the sense defined in Section 1, To show that $f$ is conjugate to a correspondence in the family (1.2) it now only remains to prove Proposition 1.4. But a holomorphic correspondence which is an equivalence relation is necessarily the covering correspondence of a rational map, and so there is a rational map $Q$ of degree three such that $(J \circ f) \cup I_{\widehat{\mathbb{C}}}=\operatorname{Cov}^{Q}$ where

$$
\operatorname{Cov}^{Q}: z \rightarrow w \quad \Leftrightarrow \quad Q(w)-Q(z)=0 .
$$

We deduce that $f=J \circ \operatorname{Cov}_{0}^{Q}$, where

$$
\operatorname{Cov}_{0}^{Q}: z \rightarrow w \quad \Leftrightarrow \quad \frac{Q(w)-Q(z)}{w-z}=0 .
$$




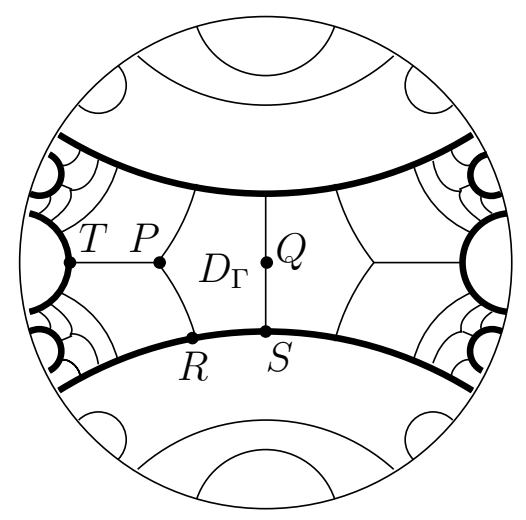

Figure 4. A Fuchsian representation of $\Gamma$. Here $P, Q, R$ and $S$ are the fixed points of $\rho, \sigma, \tau$ and $\sigma \rho \tau$. The heavy lines indicate the boundary of $D_{\Gamma_{1}}$.

Counting singular points of $f$ now tells us that $Q$ has one double and two single critical points, and that therefore up to pre- and post-compositions by Möbius transformations $Q$ is the polynomial $Q(z)=z^{3}-3 z$. It follows that up to conjugacy we may write $f$ in the form

$$
z \rightarrow w \quad \Leftrightarrow \quad(J w)^{2}+(J w) z+z^{2}=3 .
$$

It is easy to see that if we apply a further conjugacy to transform $J$ to the involution $J(z)=-z$, the equation defining the correspondence $f$ becomes a member of the family (1.2). This completes the proof of Proposition 1.4

2.3. A group-theoretic description of $\Omega$ for a mating. We shall be pinching unions of arcs in $\Omega(f)$ which are lifts of simple closed curves in the grand orbit space $\Omega(f) / f$, where $f$ is one of the matings provided by Theorem 1.3. With a view to describing these arcs, we examine the structure of $\Omega(f)$ and its relationship with $\Omega(G)$. Our first step will be to find a Fuchsian uniformisation for $\Omega(G) / G$.

Let $\Gamma$ denote the abstract group $\left\langle\sigma, \rho, \tau: \sigma^{2}=\rho^{3}=\tau^{2}=(\sigma \rho \tau)^{2}=1\right\rangle$.

Let $\mathcal{F}$ denote the moduli space of conjugacy classes of faithful discrete cocompact representations of $\Gamma$ in $P S L_{2}(\mathbb{R})$ (recall that a Fuchsian group is said to be co-compact if the quotient of the Poincare disc by its action is compact). An example of a representation of $\Gamma$ which lies in $\mathcal{F}$ is illustrated in Figure 4 Let $\mathcal{F}_{0}$ denote the path-component of $\mathcal{F}$ containing the representation illustrated. Thus a faithful discrete representation of $\Gamma$ lies in $\mathcal{F}_{0}$ if and only if there is a fundamental domain $D_{\Gamma}$ for $\Gamma$ isotopic to that illustrated in Figure 4, with boundary passing through the fixed points of the corresponding elements of $\Gamma$, in the same order but with the intervening boundary segments no longer necessarily geodesic.

Let $\sigma^{\prime}=\rho \tau \sigma$. Then $\sigma^{\prime}, \rho$ and $\tau$ together generate $\Gamma=\langle\sigma, \rho, \tau\rangle$, and satisfy the same relations. Changing to the new generating set amounts to applying an (outer) automorphism, which we denote $\beta$, to $\Gamma$. Let $\psi$ be the automorphism of $\mathcal{F}_{0}$ induced by composing the representation $\gamma \rightarrow P S L_{2}(\mathbb{R})$ with $\beta$ and replacing the boundary of $D_{\Gamma}$ in Figure 3 with that given by moving $S$ up to $Q$ (the fixed point of $\sigma^{\prime} \rho \tau$ ), and $Q$ up to $\sigma(S)$ (the fixed point of $\sigma^{\prime}$ ), but keeping $P$ and $R$ unchanged. 
Recall our description in Section 2.1 of the universal cover $\widetilde{\mathcal{D}^{o}}$ of the space $\mathcal{D}^{o}$ of conjugacy classes of faithful discrete representations of $C_{2} * C_{3}$ in $P S L_{2}(\mathbb{C})$ having connected ordinary set.

Proposition 2.5. There is a homeomorphism $\Psi: \widetilde{\mathcal{D}^{o}} \rightarrow \mathcal{F}_{0}$, which carries $t_{1 / 4}$ to $\psi$ and hence induces homeomorphisms:

(i) $\mathcal{D}^{o} \rightarrow \mathcal{F}_{0} /\left\langle\psi^{2}\right\rangle$;

(ii) $\mathcal{D}^{o} / \iota \rightarrow \mathcal{F}_{0} /\langle\psi\rangle$.

Proof. By Lemma 2.1] a point of $\widetilde{\mathcal{D}}^{o}$ corresponds to an element of $\tilde{\mathcal{S}}$, that is to say a sphere equipped with a complex structure having cone points $P$ of angle $2 \pi / 3$, and $Q, R$ and $S$ all of angle $\pi$, together with an isotopy class of paths $P R, R S$ and $S Q$. Obviously it suffices to define a homeomorphism between $\tilde{\mathcal{S}}$ and $\mathcal{F}_{0}$.

To do this we uniformise each marked orbifold $\Sigma \in \tilde{\mathcal{S}}$ as a quotient of the Poincaré disc $\Delta$ by isometries. The marked $\operatorname{arcs}$ on $\Sigma$ lift to a union of arcs, tiling $\Delta$ by translates of a polygon isotopic to that labelled $D_{\Gamma}$ in Figure 4 . The group of covering transformations of the projection from $\Delta$ to $\Sigma$ is isomorphic to $\Gamma$ by Poincaré's polygon theorem [1]. Conversely, given a faithful discrete representation of $\Gamma$ lying in $\mathcal{F}_{0}$, its quotient orbifold $\Sigma$ is an element of $\tilde{\mathcal{S}}$. Thus we have a bijection $\tilde{\mathcal{S}} \rightarrow \mathcal{F}_{0}$ which, by construction, is continuous and has a continuous inverse. Since $t_{1 / 4}$ and $\psi$ have identical effects on $\Sigma$, our composite homeomorphism $\Psi: \widetilde{\mathcal{D}^{o}} \rightarrow \mathcal{F}_{0}$ carries $t_{1 / 4}$ to $\psi$, and the assertions (i) and (ii) are immediate corollaries.

Remark 2.6. The question of finding explicit formulae for bijections between moduli spaces of representations of Kleinian groups and Fuchsian groups, such as the bijection provided by Proposition 2.5 is in general highly non-trivial, a classical example being to relate each Schottky group to a Fuchsian group representing the same surface.

Now let $\Gamma_{1} \subset \Gamma$ be the subgroup generated by $\rho \tau$ (which has infinite order), the involution $\rho^{-1} \tau \rho$, and all involutions of the form $W \rho^{-1} \tau \rho W^{-1}$, where $W$ runs through those words in $\sigma$ and $\rho$ which have rightmost letter $\sigma$. Then $\Gamma_{1}$ has as fundamental domain the region $D_{\Gamma_{1}}$ bounded by heavy lines in Figure 4. Note that $D_{\Gamma_{1}} / \Gamma_{1}$ is a topological cylinder, the top edge of the region $D_{\Gamma_{1}}$ in Figure 4 being identified with the bottom edge, each of the arcs on the left-hand edge being folded in onto an interval, and each of the arcs on the right-hand edge also being folded in onto an interval.

Suppose $f$ is a $2: 2$ holomorphic correspondence which is a mating, constructed as in Theorem 1.3. between a faithful discrete representation of $C_{2} * C_{3}$ in $P S L_{2}(\mathbb{C})$ having connected ordinary set and a quadratic map $z \rightarrow z^{2}+c$ having connected Julia set. Let $\Gamma \subset P S L_{2}(\mathbb{R})$ be the Fuchsian representation associated to it by Proposition 2.5, and let $\Gamma_{1}$ be the subgroup of $\Gamma$ defined above.

Proposition 2.7. There is a bi-analytic homeomorphism

$$
D_{\Gamma_{1}} / \Gamma_{1} \cong \Delta / \Gamma_{1} \rightarrow \Omega(f)
$$

carrying the action of the pair $\left\{\sigma \rho, \sigma \rho^{-1}\right\}$ on $D_{\Gamma_{1}} / \Gamma_{1}$ to that of the correspondence $f$ on $\Omega(f)$. 
Proof. From the construction of the mating $f$ in our outline proof of Theorem 1.3 (in Section 2.2), it is apparent that $\left(\Delta, \Gamma_{1}\right)$ uniformises $\Omega(f)$ : the set $D_{\Gamma_{1}} \cup \rho D_{\Gamma_{1}} \cup$ $\rho^{-1} D_{\Gamma_{1}}$ in Figure 4, when quotiented by the boundary identifications induced by $\Gamma_{1}$, becomes the annulus $B$ of Figure 3, and the maps $\sigma \rho$ and $\sigma \rho^{-1}$ become the two 'branches' of the correspondence $f$ on $\Omega(f)$.

Corollary 2.8. A mating between $q_{c}$ and $r \in \mathcal{D}^{o}$ constructed by the method of Theorem 1.3 is canonically isomorphic to a mating between $q_{c}$ and $\iota(r)$.

Proof. The outer automorphism $\beta$ defined by replacing the generator $\sigma$ of $\Gamma$ by $\sigma^{\prime}=$ $\rho \tau \sigma$ stabilises $\Gamma_{1}$, and the correspondence induced by $\left\{\sigma \rho, \sigma \rho^{-1}\right\}$ on $\Delta / \Gamma_{1}$ is the same as that induced by $\left\{\sigma^{\prime} \rho, \sigma^{\prime} \rho^{-1}\right\}$, since $\sigma^{\prime} \rho=\rho \tau \sigma \rho$ and $\sigma^{\prime} \rho^{-1}=\rho \tau \sigma \rho^{-1}$.

Remark 2.9. The idea of regarding $\Omega(f)$ as a quotient of $\Delta$ by an infinitely generated Fuchsian group is originally due to Chris Penrose.

Remark 2.10. We can recover the action of the Kleinian group $G=\langle\sigma, \rho, \chi\rangle$ on $\Omega(G)$ from the action of the corresponding Fuchsian group $\Gamma=\langle\sigma, \rho, \tau\rangle$ on $\Delta$, as follows. Take the polygon $D_{\Gamma_{2}}=D_{\Gamma_{1}} \cup \rho \tau\left(D_{\Gamma_{1}}\right)$ formed by two copies of $D_{\Gamma_{1}}$, one above the other, identify the top and bottom edges of this polygon to form a cylinder, then fold and glue the left-hand edge together and fold and glue the right-hand edge together, to form a sphere. The quotient $D_{\Gamma_{2}} / \sim$, which can also be described as an orbit space $\Delta / \Gamma_{2}$ for an appropriate infinitely generated subgroup $\Gamma_{2} \subset \Gamma$, is conformally equivalent to $\Omega(G)$. Indeed $\Gamma_{2} \cong \pi_{1}(\Omega(G)$ ), and the projection $\Delta \rightarrow \Delta / \Gamma_{2}$ is the universal cover for $\Omega(G)$. Under the bijection from $D_{\Gamma_{2}} / \sim$ to $\Omega(G)$ the ends of $D_{\Gamma_{2}}$ (the cusps) become the points of the limit set $\Lambda(G)$ of the action of the Kleinian group $G$ on $\hat{\mathbb{C}}$.

\section{The PINCHING DEFormation}

3.1. The arcs to be pinched. To describe the arcs that we shall pinch later, we first fix a standard faithful discrete representation $r_{*}$ of $C_{2} * C_{3}$ having connected ordinary set, and a path $l$ from a fixed point $R$ of $\chi \rho$ to a fixed point $S$ of $\chi \sigma$ (so $R$ and $S$ are as illustrated in Figure 21). For convenience we may choose $r_{*}$ and $l$ so that the corresponding group $\Gamma$ has the reflection symmetry in the horizontal axis apparent in Figure 4. Now consider the double cover $\tilde{\Sigma}$ of the orbifold $\Sigma$ ramified at all four cone points. This is a torus, with a single cone point $P$ of angle $4 \pi / 3$, represented by the central hexagon $D_{\Gamma} \cup \sigma D_{\Gamma}$ illustrated in Figure 4 with the top edge identified with the bottom edge, and the left-hand edge identified with the right-hand edge. While $\tilde{\Sigma}$ is not itself a quotient of the unit disc $\Delta$ by a subgroup of $P S L_{2}(\mathbb{C})$ (since the cone point is not of angle $2 \pi / n$ ), nevertheless we may equip $\tilde{\Sigma}$ with the metric induced by the restriction of the hyperbolic metric on $\Delta$ to the hexagon $D_{\Gamma} \cup \sigma D_{\Gamma}$. The involution $\sigma$ (on $\Delta$ ) induces an involution $\tilde{\sigma}$ on $\tilde{\Sigma}$ such that $\tilde{\Sigma} / \tilde{\sigma}=\Sigma$.

Lemma 3.1. For each rational number $p / q$ there is a geodesic arc $\delta_{p / q}$ in $\Sigma$ which has end points two of the three cone points of angle $\pi$, which misses the other cone point of angle $\pi$ and the cone point of angle $2 \pi / 3$, and which has lift $\tilde{\delta}_{p / q}$ to $\tilde{\Sigma} a$ simple closed geodesic of winding number $p / q$.

Proof. For each such $p / q$ (in lowest terms), there is a simple closed curve of winding number $p / q$ on the torus $\tilde{\Sigma}$, passing through (i) the cone points $Q$ and $S$ if $q$ is 

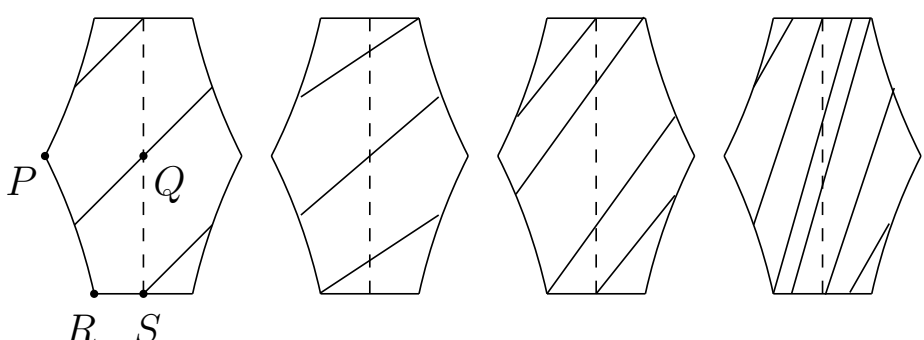

FIgURE 5 . The $\operatorname{arcs} \tilde{\delta}_{p / q}$ for $p / q=1 / 2,1 / 3,2 / 3$ and $4 / 3$, respectively.

even, (ii) the cone points $Q$ and $R$ if $p$ and $q$ are both odd, and (iii) the cone points $R$ and $S$ if $p$ is even. Examples are illustrated in Figure 5 for typical cases of each type.

Note that when we add an even integer to $p / q$, the new $\delta_{p / q}$ is an arc between the same two cone points on $\Sigma$. But when we add an odd integer, the roles of $Q$ and $S$ are interchanged.

In every case the simple closed curve on $\tilde{\Sigma}$ can be chosen to be invariant under $\tilde{\sigma}$. Since it passes through the lifts of two cone points, it descends to an arc on $\Sigma$ joining these two points. We define $\delta_{p / q}$ to be a representative of shortest length in the isotopy class of this arc, relative to its end points and the other two cone points on $\Sigma$. Note that there must exist such a minimal length example, as arcs which pass through one or both of the other cone points have lengths which are local maxima (since all the cone points have cone angle less than $2 \pi$ ).

Let $A_{p / q}$ denote the lift of $\delta_{p / q}$ to the cylinder $\left(D_{\Gamma} \cup \sigma D_{\Gamma}\right) / \Gamma_{1}$ constructed by identifying the top and bottom of the hexagon. Thus $A_{p / q}$ consists of $q$ arcs each running from one boundary circle of this cylinder to the other. Consider the union $\Gamma A_{p / q}$ of all lifts of $\delta_{p / q}$. Recall that $D_{\Gamma_{1}} / \Gamma_{1}$ is a cylinder, with ends corresponding to $\partial \Lambda_{-}$and $\partial \Lambda_{+}$(by Proposition 2.7), that the correspondence $f$ acts on $\partial \Lambda_{-}$as a quotient of the doubling map, and that $f^{-1}$ acts on $\partial \Lambda_{+}$as a quotient of the doubling map. For simplicity of description assume that $\partial \Lambda_{-}$is a topological circle and the action of $f$ on it is that of the doubling map (this is the case when the quadratic map in the mating corresponds to a value of $c$ in the interior of the main cardioid of the Mandelbrot set): obvious adaptations are possible for the cases where $\partial \Lambda_{-}$is a proper quotient of the circle.

If we label the ends of $\partial D_{\Gamma_{1}}$ by binary sequences as indicated in Figure 6, then the folding identifications induced by $\Gamma_{1}$ impose the usual quotient from the space of binary sequences to the unit circle, carrying the shift to the doubling map. Thus, under our assumption that $\partial \Lambda_{-}$is the circle, points of $\partial \Lambda_{-}$are labelled (Figure 6) in such a way that $f^{-1}: \partial \Lambda_{-} \rightarrow \partial \Lambda_{-}$(a $1: 2$ correspondence) is defined by "right shift and insert 0 or 1 " according as the branch of $f^{-1}$ is $\rho \sigma$ or $\rho^{-1} \sigma$ respectively, and points of $\partial \Lambda_{+}$are labelled in such a way that $f: \partial \Lambda_{+} \rightarrow \partial \Lambda_{+}$(also a $1: 2$ correspondence) is defined by "right shift and insert 0 or 1" according as the branch of $f$ is $\sigma \rho$ or $\sigma \rho^{-1}$, respectively. We adopt the usual notational convention that a bar over a symbol (or group of symbols) indicates the infinite repetition of that symbol (or group of symbols). 


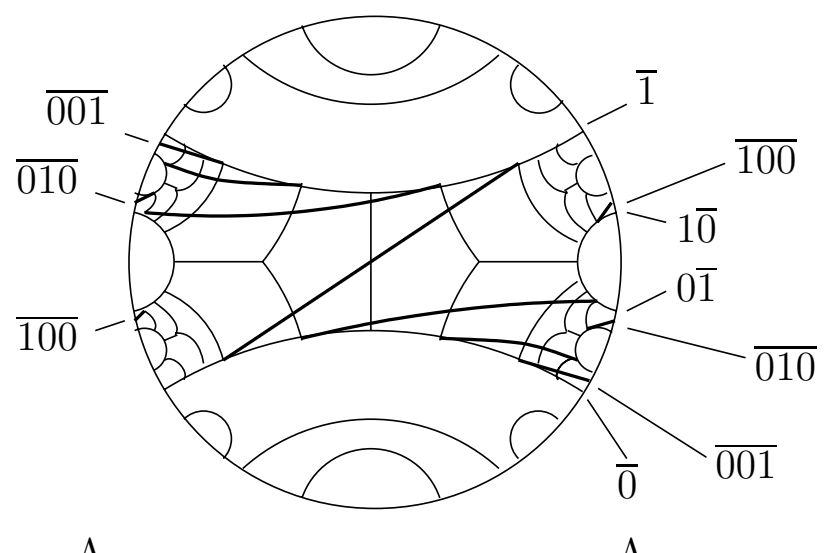

$\Lambda_{-}$

$\Lambda_{+}$

FiguRE 6 . The three arcs linking $\Lambda_{-}$to $\Lambda_{+}$in the case $p / q=1 / 3$ (the other images of these arcs under $\Gamma$ are not shown).

Definition 3.2. An infinite sequence of 0's and 1's is known as Sturmian if the binary number it represents on the circle has as its orbit under the doubling map a sequence of points arranged in the same order around the circle as for a rigid rotation.

One may assign a rotation number to each Sturmian sequence $s$, namely the limit as $n$ tends to infinity of the proportion of the first $n$ digits of $s$ which are 1's, or equivalently the rotation number of the rigid rotation having orbit points in the same order as those of $s$. Note that such a rotation number is only defined mod 1 . For each rational $p / q(\bmod 1)$ there is a unique periodic Sturmian orbit of rotation number $p / q$ (this was observed by Morse and Hedlund, who introduced the notion of Sturmian sequences). We remark that the points of each periodic Sturmian orbit $\mathcal{O}$ must be contained in an interval of length less than $1 / 2$ on the circle $\mathbb{R} / \mathbb{Z}$, as the doubling map must preserve the cyclic order of $\mathcal{O}$ (see [6] for more about this and other properties of Sturmian sequences).

Example 3.3. The infinite sequences $\overline{01}, \overline{001}$ and $\overline{00101}$ are Sturmian, of rotation numbers $1 / 2,1 / 3$ and $2 / 5$, respectively.

Proposition 3.4. $\left(\Gamma A_{p / q} \cap D_{\Gamma_{1}}\right) / \Gamma_{1}$ contains exactly $q$ arcs which join $\Lambda_{-}$to $\Lambda_{+}$. These land on $\partial \Lambda_{-}$at points of the unique Sturmian orbit of rotation number $p / q$ $(\bmod 1)$ of the $2: 1 \operatorname{map} f: \partial \Lambda_{-} \rightarrow \partial \Lambda_{-}$and at the other end they land on $\partial \Lambda_{+}$ at points of the unique Sturmian orbit of $f^{-1}$ of rotation number $p / q(\bmod 1)$.

Proof. The fact that there are exactly $q$ arcs joining $\Lambda_{-}$to $\Lambda_{+}$follows at once from the fact that exactly $q$ arcs in $\left(\Gamma A_{p / q} \cap D_{\Gamma_{1}}\right) / \Gamma_{1}$ cross the equator circle of the central cylinder $\left(D_{\Gamma} \cup \sigma D_{\Gamma}\right) / \Gamma_{1}$ (the vertical line in the central hexagon in $\left.D_{\Gamma_{1}}\right)$. The action of the correspondence $f^{-1}=\left\{\rho^{-1} \sigma, \rho \sigma\right\}$ on these arcs is to map the $j$ th arc to the $(j+p)$ th arc for each $j$, where the arcs are counted modulo $q$, from the bottom of the central hexagon upwards. Thus the action of $f^{-1}$ on the landing point of the $j$ th arc on $\Lambda_{+}$is to send it to the landing point of the $(j+p)$ th arc, for each $j$. Similarly $f$ sends the $j$ th landing point on $\Lambda_{-}$to the $(j+p)$ th. 
Definition 3.5 (of the $\operatorname{arc} \gamma_{p / q}$ ). For each $p / q$ we pick as $\gamma_{p / q}$ one of the $q$ components of $\left(\Gamma A_{p / q} \cap D_{\Gamma_{1}}\right) / \Gamma_{1}$ which cross the equator circle of the central cylinder and therefore join $\Lambda_{-}$to $\Lambda_{+}$. For definiteness, when $q$ is odd we take $\gamma_{p / q}$ to be the component which passes through $R$ (the fixed point of $\tau$ ) and when $q$ is even we take it to be the component which passes through $S$ (the fixed point of $\sigma \rho \tau$ ). We remark that in the case $p / q=0$ there is just one component crossing the vertical symmetry line of the central hexagon, and it passes through both of these points.

In Figure 6 we illustrate $\gamma_{1 / 3}$, which joins $\overline{010} \in \Lambda_{-}$to $\overline{100} \in \Lambda_{+}$, and its two images which also join $\Lambda_{-}$to $\Lambda_{+}$. These join $\overline{100} \in \Lambda_{-}$to $\overline{010} \in \Lambda_{+}$, and $\overline{001} \in \Lambda_{-}$ to $\overline{001} \in \Lambda_{+}$, respectively. Arcs $\gamma_{(3 n+1) / 3}$ for values of $n$ other than 0 , and their images, join the same pairs of points in $\Lambda_{-}$and $\Lambda_{+}$, but wind a different number of times around the cylinder $D_{\Gamma_{1}} / \Gamma_{1}$.

For general rational $p / q$ we have the following:

Algorithm 3.6. Each point in $\Lambda_{-}$represented by a Sturmian $p / q$ word $\overline{u_{1} \ldots u_{q}}$ is joined (by $\gamma_{p / q}$ or one of its images) to the point in $\Lambda_{+}$represented by the Sturmian $p / q$ word $\overline{u_{q-1} u_{q-2} \ldots u_{1} u_{q}}$.

Proof. Both $\sigma \rho$ and $\sigma \rho^{-1}$ map the fixed point $P$ of $\rho$ to $\sigma P$. It follows that $f$ maps the pair of geodesics landing on $\Lambda_{-}$either side of $\overline{1}$ to the pair of geodesics landing on $\Lambda_{+}$either side of $\overline{1}$ (Figure 6). The pair of landing points either side of $\overline{1}$ are represented by the maximum and minimum Sturmian $p / q$ words, $M_{p / q}$ and $m_{p / q}$ respectively, so the arcs landing at these points of $\Lambda_{-}$have their opposite ends at the points of $\Lambda_{+}$represented by $s\left(m_{p / q}\right)$ and $s\left(M_{p / q}\right)$ respectively, where $s$ denotes left shift (i.e. 'forget the first digit'). Since it is easily proved from the staircase algorithm for Sturmian words [6] that the minimum word $m_{p / q}=\overline{v_{q} \cdots v_{1}}$ is the reverse of the maximum word $M_{p / q}=\overline{v_{1} \ldots v_{q}}$, the result follows. Indeed we may regard the $q$ arcs joining $\Lambda_{-}$to $\Lambda_{+}$as indexed by a marked digit in a bi-infinite Sturmian word, and the action of $f$ and $f^{-1}$ on these arcs as moving the marker left and right.

Remark 3.7. Which two of the three cone points on $\Sigma$ of cone angle $\pi$ are the end points of the $\operatorname{arc} \delta_{p / q}$ is determined by the reflection symmetries of the biinfinite periodic Sturmian word of rotation number $p / q$ mod 1. Each such word has reflection symmetries of exactly two of four possible types: reflection at a 0 , or at a 1, or between two adjacent 0's or 1's. Which two types occur depends on whether (after reduction of $p / q \bmod 1) p$ is even, $q$ is even, or $p$ and $q$ are both odd. For example the bi-infinite word generated by $\overline{00101}$, a case where $p$ is even, has reflection points between the first two 0's and at the third 0. The stabiliser of any lift of $\delta_{p / q}$ to $\Delta$ is an infinite dihedral group, generated by a pair of involutions fixing adjacent lifts of cone points on the arc, and indeed isomorphic to the group of symmetries of the bi-infinite periodic Sturmian word.

Remark 3.8. The same construction of geodesic arcs crossing the central hexagon can be followed through for irrational slope $\nu$ in place of $p / q$. One then obtains a lamination on $D_{\Gamma_{1}} / \Gamma_{1}$, with singular leaves passing through the fixed point of $\rho$ and its translates. In this case the leaves crossing the hexagon join a Cantor set in $\partial \Lambda_{-}$, the unique closed invariant Sturmian set of rotation number $\nu \bmod 1$, to the analogous Cantor set in $\partial \Lambda_{+}$. The algorithm above also applies in this case to tell us which points are joined to which; we omit details here. 


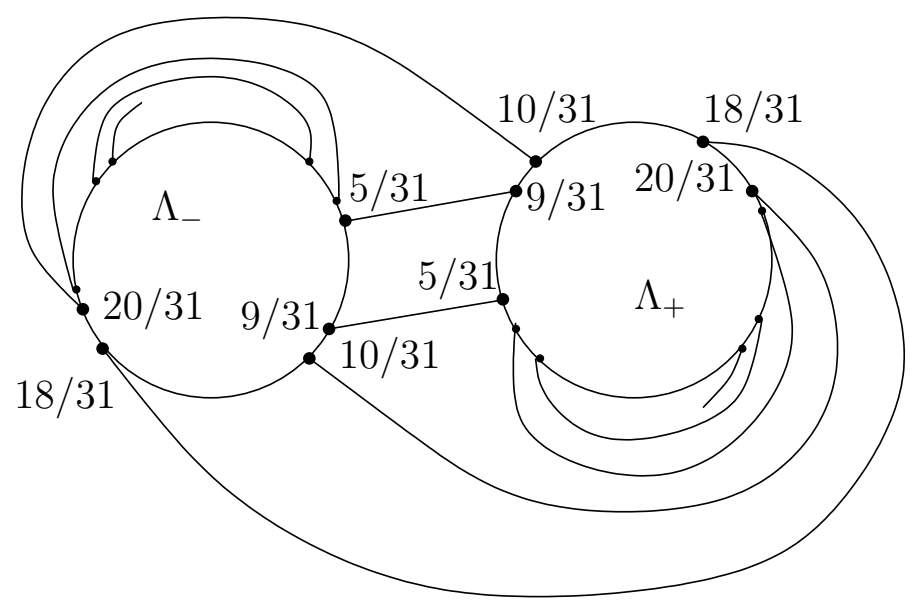

Figure 7 . The Sturmian orbits of rotation number $2 / 5$ on $\Lambda_{-}$and $\Lambda_{+}$, the five arcs joining them, and the first images of these under the correspondence and its inverse (subsequent images are not shown).

It remains to describe the grand orbit of $\gamma_{p / q}$ under the correspondence $f$.

We start with the special case $p / q=0$. The arc $\gamma_{0}$ is the lower boundary component of the region $D_{\Gamma_{1}}$ in Figure 4. Under $f$ this component maps to itself and to the boundary component of $D_{\Gamma_{1}}$ which passes through the point $\sigma(T)$. The grand orbit of $\gamma_{0}$ under $f$ is the union of all the boundary components of $D_{\Gamma_{1}}$, and quotienting by $f$, or equivalently by $\Gamma_{1}$, folds all these components (except the original one) into "spikes".

We now turn to general $p / q$. From the explicit construction of matings in Section 2.2 it follows that the branch of $f$ mapping $\Lambda_{-}$to $\Lambda_{+}$is defined as follows: given a word $W$ in 0 's and 1's representing a point in $\partial \Lambda_{-}$the $f$-image in $\partial \Lambda_{+}$ of that point is represented by the word $\phi(W)$ obtained by changing the parity of the first digit of $W$. It is now a straightforward computation that when $q$ is even the set of $q$ arcs joining $\Lambda_{-}$to $\Lambda_{+}$is mapped two-to-one by this branch to a set of $q / 2$ "concentric" arcs connecting pairwise the $q$ points of $\Lambda_{+}$obtained by applying the operation $\phi$ to the Sturmian $p / q$ orbit (i.e. the points of the circle opposite to points of the Sturmian orbit). When $q$ is odd, the set of $q$ arcs joining $\Lambda_{-}$to $\Lambda_{+}$ is mapped by this branch of $f$ to a set of $(q-1) / 2$ concentric arcs together with an innermost spike (Figure 7) which lands on $\Lambda_{+}$at a single point, the point opposite to the middle point of the Sturmian $p / q$ orbit. This spike arises from the fact that for $q$ odd the geodesic $\gamma_{p / q}$ passes through the fixed point of the involution $\tau$. Hence its image under the branch of $f$ we are considering passes through the fixed point of an involution in the group $\Gamma_{1}$. This fixed point is on the boundary of $D_{\Gamma_{1}}$ (indeed in Figure 4 it is the point $\sigma(T)$ ), and becomes the end point of a spike in the quotient $D_{\Gamma_{1}} / \Gamma_{1} \cong \Omega(f)$.

Applying $f$ again arbitrarily many times to our "concentric" set of $q / 2 \operatorname{arcs}$ (or $(q-1) / 2$ arcs plus a spike, if $q$ is odd), we obtain smaller and smaller copies around $\partial \Lambda_{+}$, and applying $\sigma$ to these copies we obtain similar copies around $\partial \Lambda_{-}$, together making up the grand orbit under $f$ of our original set of $q$ arcs. 
3.2. Matings between $q_{0}$ and circle-packing representations of $C_{2} * C_{3}$. We can now define precisely what we mean by the mating between $q_{0}$ and $r_{p / 2 q}$ referred to in the statement of Theorem 1.8. After the arcs which make up the grand orbit of $\gamma_{p / q}$ have been pinched, the intersection $\Lambda_{+} \cap \Lambda_{-}$is no longer empty, but consists of the $p / q$ Sturmian orbit of the correspondence on $\partial \Lambda_{+}$, identified with the same orbit (in the opposite direction) on $\partial \Lambda_{-}$. The set $\Omega$ for the pinched correspondence has $q$ components whose boundaries meet this orbit. These form what we call the principal cycle of components of $\Omega$. Together with $\Lambda_{-} \cap \Lambda_{+}$itself, they separate the Riemann sphere into two parts, one containing $\Lambda_{-} \backslash\left(\Lambda_{+} \cap \Lambda_{-}\right)$ and the other containing $\Lambda_{+} \backslash\left(\Lambda_{+} \cap \Lambda_{-}\right)$. The stabiliser (under the iterated pinched correspondence) of each of the components of the principal cycle is a group, since these components do not contain "fold" points. Moreover, it is not hard to see that this group is isomorphic to $C_{2} * C_{3}$.

Definition 3.9. A holomorphic correspondence is said to be a mating between $r_{p / 2 q}$ and $q_{0}$ if it is topologically conjugate to a correspondence obtained by pinching to a point each component of the grand orbit of $\gamma_{p / q}$ for a mating between $r_{*}$ and $q_{0}$, and if moreover the action of the stabiliser of each component of the principal cycle of the correspondence is conformally conjugate to the action of $P S L_{2}(\mathbb{Z})$ on the upper half-plane.

In a mating between $q_{0}$ and $r_{p / 2 q}$, the sets $\Lambda_{+}$and $\Lambda_{-}$are no longer copies of $K\left(q_{0}\right)$ (the unit disc) but are now each homeomorphic to a quotient $K\left(q_{0}\right)_{p / q}$ of $K\left(q_{0}\right)$ by an equivalence relation $\sim_{p / q}$ on $\partial K\left(q_{0}\right)$ (the unit circle) which may be described as follows. Let $\omega_{p / q}^{\prime}$ denote the points of the circle opposite to points of the Sturmian $p / q$ orbit $\omega_{p / q}$, so $\omega_{p / q}$ and $\omega_{p / q}^{\prime}$ are contained in disjoint intervals. To define the relation $\sim_{p / q}$ we identify the 'outermost' pair of points of $\omega_{p / q}^{\prime}$, and similarly we identify the next pair of points from the outside, and so on, folding the points of $\omega_{p / q}^{\prime}$ together in pairs. Similarly we identify in pairs the corresponding inverse images of points of $\omega_{p / q}^{\prime}$ under the doubling map, and repeat so that the relation $\sim_{p / q}$ becomes invariant under this inverse.

Remark 3.10. The justification for describing the construction in the definition as "a mating between $q_{0}$ and $r_{p / 2 q}$ " is two-fold. Firstly, both the construction and $r_{p / 2 q}$ are obtained by pinching the same simple closed curve $\delta_{p / q}$ on the same orbifold $\Sigma$, and secondly the definition agrees with our earlier definition for a mating between $q_{0}$ and the modular group. However, when $p / q \notin \mathbb{Z}$, the most direct relationship we know of between $\Omega\left(r_{p / 2 q}\right)$ and $\Omega(f)$ for the correspondence pinched along $\gamma_{p / q}$ is that given by pinching $\delta_{p / q}$ in the Fuchsian picture of $\Omega\left(r_{*}\right)$, described in Remark 2.10

Remark 3.11. Corollary 2.8 implies that a mating between $q_{0}$ and $r_{p / 2 q}$ is isomorphic to a mating between $q_{0}$ and $r_{(p+q) / 2 q}$. For example a mating between $q_{0}$ and $r_{1 / 2}$ is isomorphic to one between $q_{0}$ and the modular group. This example is easily understood directly, since $r_{1 / 2}$ is the faithful discrete representation of $C_{2} * C_{3}$ for which the limit set is a single round circle, like $P S L_{2}(\mathbb{Z})$, but for which the generator $\sigma$ of $C_{2}$ acts by interchanging the two components of the complement. We remark that $r_{p / 2 q}$ and $r_{(p+q) / 2 q}$ always have the same limit set, since the second representation is obtained from the first by composing with an (outer) automorphism of $C_{2} * C_{3}$. 
3.3. Invariant collar neighbourhoods of arcs. For the proofs of Theorem 1.6 and Theorem 1.8 we shall need well-behaved neighbourhoods of our arcs on which to support the pinching deformations. We define an invariant collar neighbourhood of an $\operatorname{arc} A$ joining $\Lambda_{-}$to $\Lambda_{+}$to be a closed set $\mathcal{N}(A)$ containing $A$, bounded by a pair of arcs joining the end points of $A$, such that under the action of $f$ the set $\mathcal{N}(A)$ has stabiliser isomorphic to the infinite dihedral group, and $\mathcal{N}(A)$ is precisely invariant under the action of this stabiliser. (Strictly speaking, $\mathcal{N}(A)$ is not a topological neighbourhood of $A$, since the end points of $A$ are on the boundary of $\mathcal{N}(A)$.)

Lemma 3.12. The arc $\gamma_{p / q}$ has an invariant collar neighbourhood.

Proof. A collar neighbourhood of each of the $q \operatorname{arcs}$ which join $\Lambda_{-}$to $\Lambda_{+}$is obtained by lifting any collar neighbourhood of the $p / q$ geodesic $\delta_{p / q}$ on the orbifold $\Sigma$. It is immediate from the action of $\sigma \rho$ and $\sigma \rho^{-1}$ on the lift of such a neighbourhood that its stabiliser under the action of $f$ is an infinite dihedral group, generated by the appropriate branch of $f^{q}$ and by $\sigma$ (which is a branch of $f^{-1} f f^{-1}$ ) composed with a branch of whichever $f^{r}$ maps the $\sigma$ image of the arc back to the arc. This lifted collar neighbourhood is precisely invariant under the action of the stabiliser.

The small copies of the $q$ arcs have collar neighbourhoods that are the images of the original collar neighbourhoods under appropriate branches of forward or backward iterates of $f$. These images are each either a bijective copy, or (in the case of a "spike") a quotient by an involution, of one of the original collar neighbourhoods. In the case of the arc $\gamma_{0}$, joining the fixed points of the doubling map on $\partial \Lambda_{-}$and $\partial \Lambda_{+}$, all the images are such quotients.

3.4. A pinching deformation. Let us consider a correspondence $p$ which represents the mating of a quadratic polynomial $q$ with a faithful and discrete representation of $C_{2} * C_{3}$ with connected ordinary set, and let $f: \Lambda_{-} \rightarrow \Lambda_{-}$be the 2 : 1-branch of $p$. We fix the curve of rotation number $p / q$ and consider its lifts $\mathcal{R}$ (for red) to $\overline{\mathbb{C}}$. Thus $\gamma=\gamma_{p / q}$ is one of the connected components of $\mathcal{R}$ which joins $\Lambda_{-}$to $\Lambda_{+}$. Let us denote its collar neighbourhood defined above by $\mathcal{N}(\gamma)$. Then $\operatorname{Stab}_{p}(\mathcal{N}(A))$ is isomorphic to the infinite dihedral group. Let $B_{-}$and $B_{+}$be both components of $\mathcal{N}(A) \backslash \gamma$.

We will first define an appropriate quasiconformal deformation on a model strip and then implement it on the dynamical plane [13].

Our model space will be a closed horizontal strip on the upper half-plane. Choose a collection of numbers $0<L_{y}<L_{r}$ (the indices $y, r$ are colours yellow and red, respectively), and then an increasing $C^{1}$-function $\tau:\left[0,1\left[\rightarrow\left[L_{r},+\infty\left[\right.\right.\right.\right.$. Let $M \subset \mathbb{R}^{2}$ be the closed subset bounded by

$$
([0,1] \times\{0\}) \cup\left(\{0\} \times\left[0, L_{r}\right]\right) \cup(\{1\} \times[0,+\infty[) \cup(\{(t, \tau(t)), t \in[0,1[\}) .
$$

Choose $v_{t}(y)$ so that $v_{t}(y)=y$ for $0 \leq y \leq L_{y}$ and that $(t, y) \mapsto\left(t, v_{t}(y)\right)$ is a $C^{1}$-diffeomorphism from $[0,1] \times\left[0, L_{r}\right] \backslash\left\{\left(1, L_{r}\right)\right\} \rightarrow M$.

We also make the following technical assumption: for any $L^{\prime}<L_{r}$, there is $\left.t\left(L^{\prime}\right) \in\right] 0,1\left[\right.$ with $t\left(L^{\prime}\right) \rightarrow 1$ as $L^{\prime} \rightarrow L_{r}$, such that for any $\left.\left.(s, y) \in\right] t\left(L^{\prime}\right), 1\right] \times\left[0, L^{\prime}\right]$, we have $v_{s}(y)=v_{t\left(L^{\prime}\right)}(y)$. Now on the straight strip $\left\{0 \leq x \leq L_{r}\right\}$, and for every $t \in[0,1]$, set

$$
\widetilde{P}_{t}(x+i y)=x+i \cdot v_{t}(y)
$$



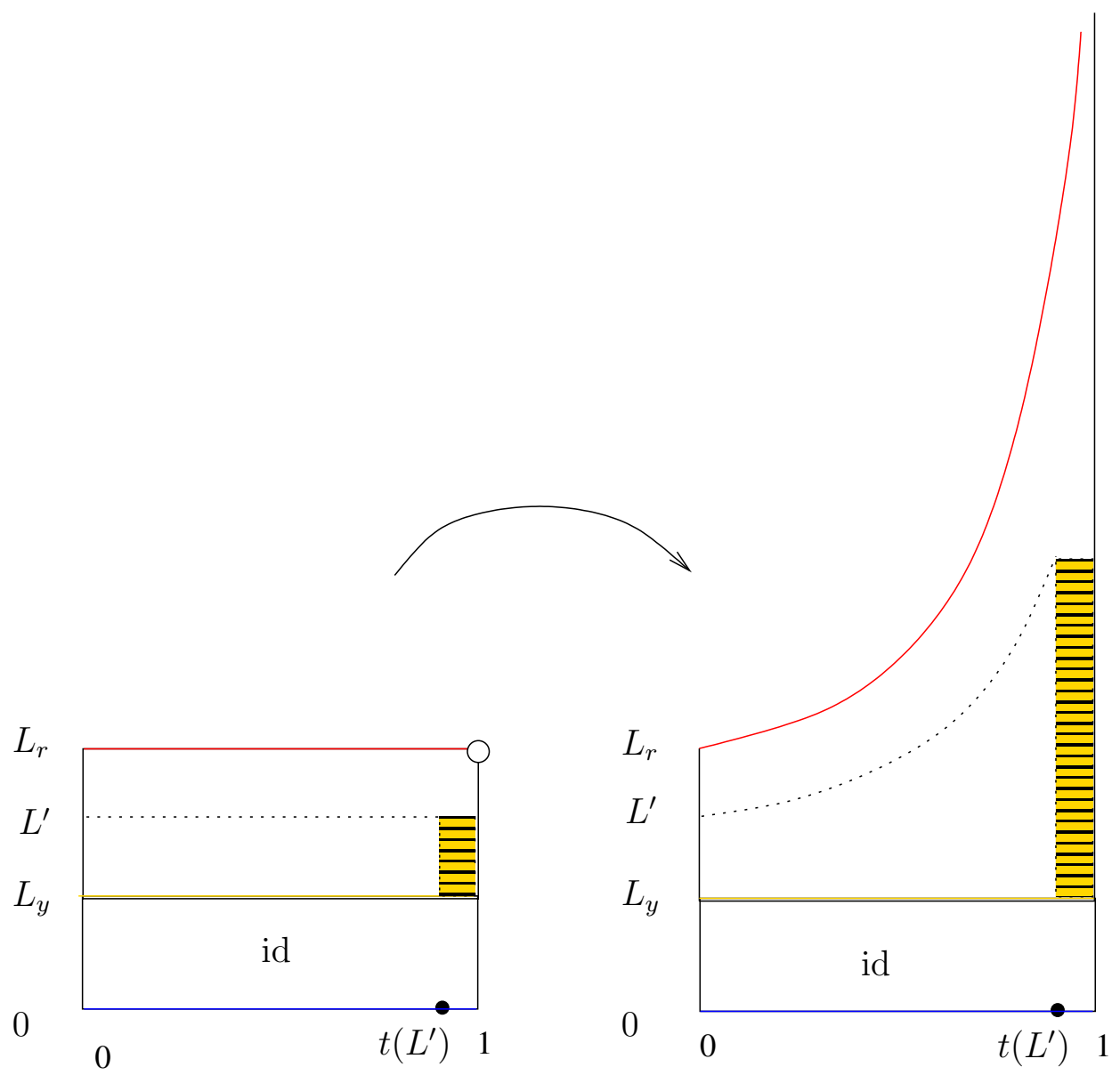

FiguRE 8. The diffeomorphism $(t, y) \mapsto\left(t, v_{t}(y)\right)$.

This map satisfies the following properties:

(1) It commutes with the translation by 1 (and by any other real number).

(2) It is the identity on the sub-strip $\left\{0 \leq y \leq L_{y}\right\}$.

(3) The coefficient of the Beltrami form

$$
\left.\frac{\partial \widetilde{P}_{t} / \partial \bar{z}}{\partial \widetilde{P}_{t} / \partial z}\right|_{x+i y}=\frac{1-\frac{\partial}{\partial y} v_{t}(y)}{1+\frac{\partial}{\partial y} v_{t}(y)}
$$

is continuous on $(t, x+i y) \in[0,1] \times\left\{0 \leq y \leq L_{r}\right\}$, whose norm is locally uniformly bounded from 1 if $(t, y) \neq\left(1, L_{r}\right)$ and tends to 1 as $(t, y) \rightarrow$ $\left(1, L_{r}\right)$.

Define conformal maps $\psi_{ \pm}: B_{ \pm} \rightarrow \mathbb{R} \times\left(0, L_{r}\right)$ which map $\gamma$ to $\mathbb{R} \times\left\{L_{r}\right\}$. For $t \in\left[0,1\left[\right.\right.$, set $\sigma_{t}^{\prime}=\left(\widetilde{P}_{t} \circ \psi_{ \pm}\right)^{*}\left(\sigma_{0}\right)$ to be the pull-back of the standard complex structure on $B_{ \pm}$. Since the action is properly discontinuous on $\Omega(f)$, we may spread $\sigma_{t}^{\prime}$ to the whole orbit of $\mathcal{N}(\gamma)$ under the correspondence $p$. We let $\sigma_{t}$ be the extension of this almost complex structure to the whole Riemann sphere by 
setting $\sigma_{t}=\sigma_{0}$ on the complement. It is a $p$-invariant complex structure. We let $\mathcal{Y}$ (for yellow) be the set of points $z$ such that $\sigma_{t}(z)$ is not the standard conformal structure for some $t$.

The family of $p$-invariant complex structures $\left(\sigma_{t}\right)_{t \in[0,1)}$ defines a pinching deformation supported on $\mathcal{R}$. We let $h_{t}$ be the quasiconformal map given by the Measurable Riemann Mapping Theorem applied to $\sigma_{t}$ normalised so that $h_{t}$ fixes both critical points of $\left.f\right|_{\Lambda_{-}}$and $\left.f^{-1}\right|_{\Lambda_{+}}$and the point at infinity as well. The correspondence $p_{t}$ defined by $p_{t}(z, w)=p\left(h_{t}^{-1}(z), h_{t}^{-1}(w)\right)$ is holomorphic by construction, and the family of pairs $\left(p_{t}, h_{t}\right)_{t \in[0,1)}$ defines a marked pinching deformation.

\section{Convergence of the Pinching Deformation}

The proofs of both Theorem 1.6 and Theorem 1.8 follow essentially the same lines. We must prove that the pinching deformation defined in the previous section converges uniformly in each case, and we must prove that in each case the limit correspondence has, as stabiliser of each of the components of the principal cycle of $\Omega$, a group conformally equivalent to $P S L_{2}(\mathbb{Z})$. The strategy for proving uniform convergence is inspired by [12, 13] where analogous statements are proved for rational maps and where detailed proofs can be found.

We proceed to prove both theorems simultaneously as far as possible. We refer to [12] and [13] when we can, instead of repeating the detailed arguments presented in these papers. The parts of the proofs which differ for the two theorems are postponed to the Sections 4.1 and 4.2. In particular, we delay the proof of the key Lemma 4.2 (stated below). The first step in the proof of the theorems is to prove that the path of quasiconformal homeomorphisms $\left(h_{t}\right)$ is equicontinuous. We will apply the following criterion, the proof of which is elementary (cf. Lemma 2.5 in [13).

Lemma 4.1 (Equicontinuity criterion at a point). Let $\mathcal{H}=\{h: \mathbb{D} \rightarrow \mathbb{C}\}$ be a family of continuous injective maps such that $\bigcup_{h \in \mathcal{H}} h(\mathbb{D})$ avoids at least 2 points in $\mathbb{C}$. Let $\left(U_{n}\right)_{n \geq 0}$ be a nested sequence of disc-like neighbourhoods of the origin in the unit disc $\mathbb{D}$ such that $A_{n}=\mathbb{D} \backslash \overline{U_{n}}$ is an annulus. If there exists a sequence $\eta_{n} \nearrow+\infty$ such that

$$
\forall h \in \mathcal{H}, \forall n \geq 0, \bmod h\left(A_{n}\right) \geq \eta_{n},
$$

then $\mathcal{H}$ is equicontinuous at the origin.

This means that we need to get infinitely many annuli with controlled moduli. The assumption on the fixed point $\beta$ will give us information on the support of the deformation: this will enable us to prove the following lemma in the respective cases.

Lemma 4.2 (One good annulus around each Julia point). Fix $r>0$.

(i) For any $x \in \partial \Lambda_{-} \cup \partial \Lambda_{+} \backslash \mathcal{R}$, there are two open neighbourhoods $N^{\prime}(x)$ and $N(x)$ of $x$ in $D\left(x, \frac{r}{4}\right)$ and $m>0$ such that $\bmod h_{t}\left(N(x) \backslash \overline{N^{\prime}(x)}\right) \geq m$ for all $t$.

(ii) For any $x=\beta_{\gamma} \in \mathcal{R} \cap\left(\partial \Lambda_{-} \cup \partial \Lambda_{+}\right)$, with $\gamma$ an $\mathcal{R}$-component, there is a sequence $\left(t_{n}\right)$ in $[0,1)$ tending to 1 , a nested sequence of annuli $\left(A_{n}\right)_{n}$ surrounding $\gamma$, and a constant $m>0$ such that $\bmod h_{t}\left(A_{n}\right) \geq m / n$ for $t \geq t_{n}$. 
Then the weak hyperbolicity condition is used to spread these annuli at every point and at every scale and therefore to imply the equicontinuity of $\left(h_{t}\right)$ (cf. the proof of the Proposition 2.3 in [13] or $\S 3$ in [12]). The estimates of the conformal moduli also enable us to analyse the structure of the fibres of any limit map and to conclude that its fibres are exactly the closures of the connected components of $\mathcal{R}$.

Any limit $h_{1}$ satisfies the conclusion of the theorem and we may also extract a convergent sequence $\left(p_{t_{n}}\right)$ of the correspondences to a correspondence $p_{1}$ (cf. Appendix A in [13).

Since the fibre structure is well understood, it follows that if there are other limits $(\widehat{h}, \widehat{p})$, then $\widehat{h} \circ h_{1}^{-1}$ defines a conjugacy which is conformal off $h_{1}\left(\partial \Lambda_{-} \cup \partial \Lambda_{+}\right)$(cf. Lemma A.2 in [13]).

Now it can be shown, as in [13, that all the limit correspondences satisfy the "weak hyperbolicity" condition on the image of $\partial \Lambda_{-} \cup \partial \Lambda_{+}$. Since $\partial \Lambda_{-} \cup \partial \Lambda_{+}$ has no interior, a standard argument of Sullivan implies that the Lebesgue measure of $h_{1}\left(\partial \Lambda_{-} \cup \partial \Lambda_{+}\right)$is zero (cf. Theorem 4.1 in [11). Furthermore, the weak hyperbolicity condition on $p_{1}$ implies that the following rigidity statement holds.

Proposition 4.3. Let $p_{0}$ and $p_{1}$ be two correspondences which are matings of weakly hyperbolic polynomials with discrete representations of $C_{2} * C_{3}$. If $p_{0}$ and $p_{1}$ are conjugate by a topological homeomorphism which is conformal off the limit sets, then the conjugacy is a Möbius transformation.

The proof of this proposition follows the same lines as Proposition 6.3 and Theorem 0.2 in [11.

Thus $\widehat{h} \circ h_{1}^{-1}$ is a Möbius transformation, whence the uniqueness of the limits $\left(p_{t}, h_{t}\right)$ as $t$ tends to 1 .

To complete the proofs of Theorem 1.6 and Theorem 1.8 it now remains only to prove Lemma 4.2 in both cases, and to prove that in each case the limit of the family of pinching deformations corresponds to the mating we are looking for.

4.1. The simple case (winding number zero). We shall make use of the statements proved in 13 for simple pinchings of rational maps, so we have to show how to get to that setting.

Using McMullen's gluing lemma (Proposition 5.5 in 16]), we may construct a rational map $R$ of degree 2 which induces a partition of the sphere $\overline{\mathbb{C}}=K \sqcup \mathcal{F}$ where $K$ is the filled-in Julia set of a quadratic-like map induced by a restriction of $R$ hybrid-equivalent to $q$, and $\mathcal{F}$ is the basin of attraction of a fixed point at infinity of multiplier $1 / 2$. For the domains of the quadratic-like map, we first choose a linearising disc $D$ for the point at infinity which contains the critical value, and set $V=\overline{\mathbb{C}} \backslash \bar{D}$. If $V^{\prime}=R^{-1}(V)$, then $R: V^{\prime} \rightarrow V$ is quadratic-like. Furthermore, we may find a forward-invariant Jordan $\operatorname{arc} \kappa$ in $\mathcal{F}$ joining the point at infinity with the corresponding $\beta$-fixed point which only cuts $\partial V$ once, and then transversally. Let $\widehat{\mathcal{R}}$ be the grand orbit of $\kappa$ for $R$. It follows that $(\widehat{\mathcal{R}} \backslash \kappa) \cap \partial V=\emptyset$.

Proposition 4.4. There is a quasiconformal $\Phi: \overline{\mathbb{C}} \rightarrow \overline{\mathbb{C}}$ such that:

- $\Phi\left(\Lambda_{-}\right)=K$ and $\Phi(\mathcal{R})=\widehat{\mathcal{R}}$

- $\Phi \circ f=R \circ \Phi$ in a neighbourhood of $\Lambda_{-}$,

- $\bar{\partial} \Phi=0$ a.e. on $\Lambda_{-}$.

Proof. We already know that there is a quasiconformal map $\phi: \overline{\mathbb{C}} \rightarrow \overline{\mathbb{C}}$ which fulfills the conclusions of the Proposition except for the condition on the curves. 
We let $U^{\prime} \Subset U$ be simply connected domains such that the extension $f: U^{\prime} \rightarrow U$ of the branch of the correspondence $f: \Lambda_{-} \rightarrow \Lambda_{-}$is a quadratic-like map hybridequivalent to $q$. It follows from the construction of $f$ that we may assume that $U$ is a fundamental domain for the involution $J$. Furthermore, we may also assume that $\phi(U)=V$.

We let $\phi_{0}: U \rightarrow V$ be a quasiconformal homeomorphism isotopic to $\phi$ rel. $\Lambda_{-}$ through an isotopy which maps $\partial U$ to $\partial V$ throughout, and such that

$$
\phi_{0}\left(\gamma_{0} \cap\left(\bar{U} \backslash U^{\prime}\right)\right)=\kappa \cap\left(\bar{V} \backslash V^{\prime}\right) \text { and }\left.R \circ \phi_{0}\right|_{\partial U^{\prime}}=\left.\phi_{0} \circ f\right|_{\partial U^{\prime}}
$$

This is possible since both sets $U \backslash \Lambda_{-}$and $V \backslash K$ are annuli and since the action of the maps $f$ and $R$ are 2:1 coverings. Define $\left(\phi_{n}\right)$ inductively so that $\phi_{n+1} \circ f=R \circ \phi_{n}$ so that $\left.\phi_{n}\right|_{\Lambda_{-}}=\left.\phi\right|_{\Lambda_{-}}$and $\left.\phi_{n}\right|_{\bar{U} \backslash U^{\prime}}=\left.\phi_{0}\right|_{\bar{U} \backslash U^{\prime}}$. This sequence is a normal family of quasiconformal mappings which admits at least one limit $\Phi: U \rightarrow V$. This map satisfies the conclusion of the proposition.

We now provide a proof of Lemma 4.2 under the assumptions of Theorem 1.6.

Proof (Lemma 4.2). We first assume that $q$ is not conjugate to $z \mapsto z^{2}+1 / 4$. Then by Lemma 2.7 in 13 we have the result we seek but for $\widehat{\mathcal{R}}$ and the rational map $R$ in place of $\mathcal{R}$ and the correspondence. By Proposition 4.4 this is all we need, except for the case of the only $\mathcal{R}$-component, $\gamma_{0}$, which is not contained in the neighbourhood $U$ of $\Lambda_{-}$. But $\gamma_{0}$ is a double cover of any other component $\gamma$ of $\mathcal{R}$ by a branch of the correspondence, and $\gamma_{0}$ has a neighbourhood which is a double cover of a disc neighbourhood of $\gamma$, by the same branch.

We now deal with $q(z)=z^{2}+1 / 4$. Let us denote by $p$ the mating of $q$ with $C_{2} * C_{3}$ and let us define $q_{0}(z)=z^{2}, p_{0}$ and $R_{0}$ the corresponding mating and rational map. We let $\left(p_{t}, \widehat{h}_{t}\right)$ be the simple pinching of $p_{0}$ considered above, and $\Phi_{0}: \Lambda_{-}\left(p_{0}\right) \rightarrow \overline{\mathbb{D}}$ be given by Proposition 4.4, It follows from Corollary 3.10 in [13] that there is a $\mu$-homeomorphism in the sense of David, $\phi: \mathbb{C} \rightarrow \mathbb{C}$, conjugating $\left.p_{0}\right|_{\Omega\left(p_{0}\right)}$ conformally to $\left.p\right|_{\Omega(p)}$. Furthermore, a constant $K_{0} \geq 1$ exists such that the set of points $z \in \mathbb{C}$ for which the dilatation ratio $K_{\phi}(z)$ is at least $K_{0}$ is contained in the disjoint union of the orbit of an invariant sector $S \subset \operatorname{int}\left(\Lambda\left(p_{0}\right)\right)$ with vertex $\beta$ (see Lemma 2.1 in 10 for details).

We claim that the image under $\phi$ of the controlled annuli for $p_{0}$ also have controlled moduli. For points outside the red set, this is because the set where $K_{\phi}$ is large is contained in the union of sectors so that the Key lemma in 13, which implies the bounds on the moduli, also holds for these domains.

For points in the red set, we must be more precise and use intermediate results which are established for the proof of Lemma 2.7 in [13. We refer to $\$ 2.5$ in [13] for the details. We let $Y$ be the connected component of $\mathcal{Y}\left(p_{0}\right)$ which contains $\gamma_{0}$. In the proof of the equicontinuity at those points, it is shown that there is a sequence $\psi_{n}: A_{n} \rightarrow(-C-(n+1), C+(n+1))^{2} \backslash[-C-n, C+n]^{2}$ of homeomorphisms, where $C$ is a fixed positive real number, such that, for $t \geq t_{n}, \psi_{n} \circ \widehat{h}_{t}^{-1}$ is uniformly quasiconformal off $\mathcal{Y} \backslash Y$. Moreover, $\psi_{n}$ maps $\Phi_{0}(S) \cap A_{n}$ onto a rectangle $Q_{n}=$ $[-C-(n+1),-C-n] \times\left[C_{1}, C_{2}\right]$ for fixed constants $C_{1}$ and $C_{2}$.

The bound on the moduli for the cauliflower map $z \mapsto z^{2}+1 / 4$ comes from a length-area argument provided by metrics $\left(\rho_{n}^{t}\right)$ defined as follows. Let $t \geq t_{n}$; on 
$\widehat{h}_{t}\left(\mathcal{Y}\left(p_{0}\right) \backslash Y\right)$, we let $\rho_{n}^{t}=0$ and on its complement we define

$$
\rho_{n}^{t}=\frac{1}{\left|\partial_{z} \widehat{h}_{t} \circ \psi_{n}^{-1}\right|-\left|\partial_{\bar{z}} \widehat{h}_{t} \circ \psi_{n}^{-1}\right|} \circ\left(\psi_{n} \circ \widehat{h}_{t}^{-1}\right) .
$$

This kind of metric is used to prove the quasi-invariance of moduli of annuli for quasiconformal maps. This metric yields the bound $\bmod \widehat{h}_{t}\left(A_{n}\right) \geq m / n$ where $m>0$ is independent of $n$.

Similarly, we let $\widehat{\rho}_{n}^{t}=0$ for points in $h_{t} \circ \phi\left(\mathcal{Y}\left(p_{0}\right) \backslash Y\right)$ and on the complement, we let

$$
\hat{\rho}_{n}^{t}=\frac{\rho_{n}^{t}}{\left|\partial_{z} \phi_{t}\right|-\left|\partial_{\bar{z}} \phi_{t}\right|} \circ \phi_{t}^{-1}
$$

where $\phi_{t}=h_{t} \circ \phi \circ \chi_{t}^{-1}$. It follows from the construction of $\phi$ that $K_{\phi} \asymp n$ on $Q_{n}$ (see Lemma 2.1 in 10]), so that the area of $h_{t}\left(\phi\left(Q_{n}\right)\right)$ is at most a multiple of $n$, as the area of $h_{t}\left(\phi\left(A_{n} \backslash Q_{n}\right)\right)$, for the metric $\widehat{\rho}_{n}^{t}$. Thus, we get $\bmod h_{t}\left(\phi_{0}\left(A_{n}\right)\right) \geq c / n$. Whence we obtain the estimates of the moduli for these points also.

The following proposition now completes the proof of Theorem 1.6 ,

Proposition 4.5. Under the assumptions of Theorem 1.6, the limit $p_{1}$ of $\left(p_{t}\right)$ is a mating of $q$ with $P S L_{2}(\mathbb{Z})$.

Proof. The limiting correspondence $p_{1}$ inherits a compatible involution $J$ from $p_{0}$, so by Proposition 1.4 this correspondence is conjugate to some member of the family (1.2), or equivalently to $J \circ C o v_{0}^{Q}$ for $Q(z)=z^{3}-3 z$ and $J$ some (Möbius) involution. The proof of the proposition now follows the same steps as the proof of Theorem 7.1 in [3], which states an analogous result for the degree 4 Chebyshev polynomial in place of $Q$. We summarise the steps, but refer the reader to [3] for technical details. The topological dynamics of $p_{1}$ ensure that there exist a transversal $D_{Q}$ for $Q$ and a fundamental domain $D_{J}$ for $J$ such that the complement of the union of the interiors of $D_{Q}$ and $D_{J}$ consists precisely of the fixed point $\Lambda_{+} \cap \Lambda_{-}$. This fixed point is parabolic for $f$ and it follows from local analysis that in a neighbourhood the boundaries of $D_{Q}$ and $D_{J}$ may be chosen to be smooth curves, tangent to one another at the fixed point. The set $D_{Q} \cap D_{J}$ is a fundamental domain for the action of $\left.f\right|_{\Omega}$, and since $\left.f\right|_{\Omega}$ and $\left.f^{-1}\right|_{\Omega}$ have no critical points (only double points) we know that $\left.f\right|_{\Omega}$ is conformally conjugate to $\left\{\sigma \rho, \sigma \rho^{-1}\right\}$ for some Fuchsian representation of $C_{2} * C_{3}$ acting on the open upper half of the complex plane. To show that this action is indeed that of $\operatorname{PSL}(2, \mathbb{Z})$ it suffices to show that in the upper half-plane the images of $\partial D_{Q}$ and $\partial D_{J}$ converge to the same point on the real axis. This can be shown to follow from the fact that $\partial D_{Q}$ and $\partial D_{J}$ are smooth curves which meet tangentially (see [3]).

4.2. Pinching arcs of non-zero rational winding number. Let $p$ be a correspondence which is a mating between $z \mapsto z^{2}$ and a faithful discrete representation of $C_{2} * C_{3}$ in $P S L_{2}(\mathbb{C})$ with connected ordinary set. In this section we prove Lemma 4.2 for curves in $\Omega(p)$ with non-zero rational rotation number. The fact that the Julia set is a quasicircle will be crucial in the proof, which closely follows the argument in $\S 3$ of [12.

The first step is to straighten the limit set and the support of the pinching. Figure 9 illustrates an example. 


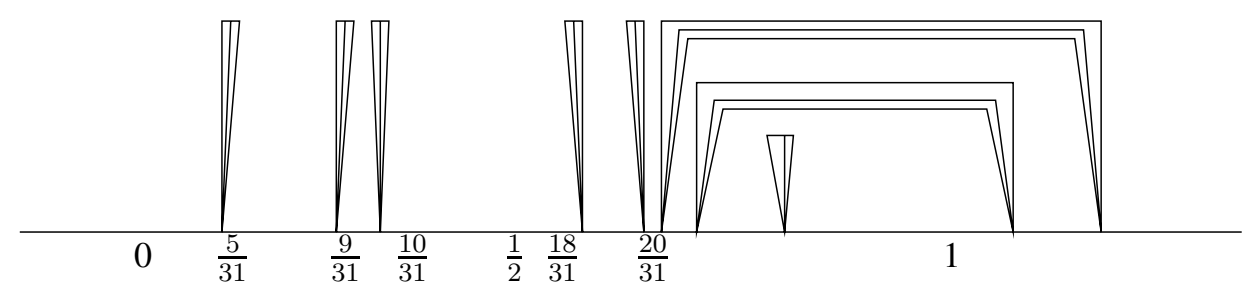

Figure 9. Image under $\chi$ of the collars of the first two generations of the orbit of $\gamma_{p / q}$, in the case $p / q=2 / 5$ (cf. Figure 7 ).

Lemma 4.6. There is a quasiconformal map $\chi: \overline{\mathbb{C}} \rightarrow \overline{\mathbb{C}}$ such that $\chi\left(\partial \Lambda_{-}\right)=S S^{1}$, which satisfies the following properties:

- $\chi$ is conformal on the interior of $\Lambda_{-}$;

- $\chi$ conjugates $f$ to $z \mapsto z^{2}$ in a neighbourhood of the interior of $\Lambda_{-}$;

- components of $\mathcal{Y}$ which are attached at two points $x$ and $y$ to $\Lambda_{-}$are mapped into rectangles in (log)-polar coordinates with base $[\chi(x), \chi(y)]$;

- components $Y$ of $\mathcal{Y}$ which are attached at a single point $x$ to $\Lambda_{-}$are mapped into sectors based at $\chi(x)$.

Proof. The restriction of $\chi$ to $\Lambda_{-}$is given by the Böttcher coordinates of $f$. The extension of $\chi$ to the outside makes use of a pull-back argument (see pp. 14-15 in [12]).

The next step of the proof is to control the moduli of many annuli. We place ourselves in the coordinates given by $\chi$. As in [12, we may define annuli bounded by rectangles in the log-polar coordinates which avoid the image of $\mathcal{Y}$ under $\chi$.

As in the case of simple pinchings, there is no problem with the curves which link both components of $\Lambda$, because they cover other components which do not. This enables us to prove Lemma 4.2 (cf. Propositions 3.3 and 3.4 in [12]).

Finally, the following proposition completes the proof of Theorem 1.8 ,

Proposition 4.7. Under the assumptions of Theorem 1.8, the limit $p_{1}$ of $\left(p_{t}\right)$ is a mating of $z \mapsto z^{2}$ with the circle-packing representation $r_{p / 2 q}$ of $C_{2} * C_{3}$.

Proof. As in the proof of Proposition 4.5, the limiting correspondence $p_{1}$ is necessarily conjugate to some member of the family (1.2), or equivalently to $J \circ \operatorname{Cov}_{0}^{Q}$ for $Q(z)=z^{3}-3 z$ and $J$ some (Möbius) involution. Once again we can now follow the same steps as in the proof of Theorem $7.1 \mathrm{in}\left[3\right.$. Transversals $D_{Q}$ and $D_{J}$ can be chosen this time such that the complement of the union of their interiors consists precisely of the period $q$ parabolic orbit $\Lambda_{+} \cap \Lambda_{-}$, and such that in a neighbourhood of any point of this orbit the boundaries of these transversals are smooth curves, tangent to one another at the orbit point. From the fact that $\Omega$ is now a countable union of topological discs and our knowledge of the topological dynamics of $f$ (using convergence of the pinching deformation), we know that $\left.f\right|_{\Omega}$ and $\left.f^{-1}\right|_{\Omega}$ have no critical points (only double points) and that for any component of $\Omega$ which meets the period $q$ orbit $\Lambda_{+} \cap \Lambda_{-}$, the iterated branches of $f$ which stabilise the component are conformally conjugate to the elements of the group generated by $\left\{\sigma \rho, \sigma \rho^{-1}\right\}$ for some Fuchsian representation of $C_{2} * C_{3}$ acting on the open upper half of the complex plane. As in the proof of Proposition 4.5, the properties of the boundaries 
of $D_{Q}$ and $D_{J}$ again ensure that this representation is indeed conformally conjugate to $P S L_{2}(\mathbb{Z})$.

\section{REFERENCES}

1. A.F. Beardon, The Geometry of Discrete Groups, Graduate Texts in Mathematics No. 91, Springer Verlag 1983. MR698777|(85d:22026)

2. L. Bers, Simultaneous uniformization, Bull. Amer. Math. Soc. 66 (1960), 94-97. MR0111834 $(22: 2694)$

3. S. Bullett and M. Freiberger, Holomorphic correspondences mating Chebyshev-like maps and Hecke groups, Ergodic Theory and Dynamical Systems 25 (2005), 1057-1090. MR 2158397

4. S. Bullett and W.J. Harvey, Mating quadratic maps with Kleinian groups via quasiconformal surgery, Electronic Research Announcements of the AMS 6 (2000), 21-30. MR 1751536 (2000m:37068)

5. S. Bullett and C. Penrose, Mating quadratic maps with the modular group, Inventiones Mathematicae 115 (1994), 483-511. MR.1262941 (95c:58148)

6. S. Bullett and P. Sentenac, Ordered orbits of the shift, square roots and the devil's staircase, Math. Proc. Cam. Phil. Soc. 115 (1994), 451-481. MR1269932 (95j:58043)

7. Cui, G.-Z., Dynamics of rational maps, topology, deformation and bifurcation, preprint, May 2002.

8. A. Douady and J.H. Hubbard, Itération des polynômes quadratiques complexes, C.R. Acad. Sci. Paris 294 (1982), 123-126. MR651802 (83m:58046)

9. A. Douady and J.H. Hubbard, On the dynamics of polynomial-like mappings, An. de l'École Norm. Sup. 18 (1985), 287-343. MR816367 (87f:58083)

10. P. Haïssinsky, Chirurgie parabolique, C.R. Acad. Sci. Paris 327 (1998), 195-198. MR 1645124 (99i:58127)

11. P. Haïssinsky, Rigidity and expansion for rational maps, J. London Math. Soc. (2) 63 (2001), no. 1, 128-140. MR.1802762 (2001m:37085)

12. P. Haïssinsky, Pincement de polynômes, Comment. Math. Helv. 77 (2002), no. 1, 1-23. MR 1898391 (2003m:37061)

13. P. Haïssinsky and Tan Lei, Matings of geometrically finite polynomials, Fund. Math. 181 (2004), 143-188. MR2070668(2005e:37106)

14. B. Maskit, On Klein's combination theorem, Trans. AMS 120 (1965), 499-509. MR0192047 $(33: 274)$

15. B. Maskit, Parabolic elements in Kleinian groups, Ann. Math. 117 (1983), 659-668. MR701259 (85a:30073)

16. C.T.McMullen, Automorphisms of rational maps, Holomorphic functions and moduli, Vol. I (Berkeley, CA, 1986), 31-60, Math. Sci. Res. Inst. Publ., 10, Springer, New York, 1988. MR 955807 (89m:58187)

17. C.T. McMullen, Cusps are dense, Annals of Mathematics 133 (1991), 217-247. MR.1087348 (91m:30058)

18. F. Przytycki and S. Rohde, Porosity of Collet-Eckmann Julia sets, Fund. Math. 155 (1998), no. 2, 189-199. MR1606527 (2000b:37047)

19. M. Rees, Realization of matings of polynomials as rational maps of degree two, manuscript, 1986.

20. Tan Lei, Matings of quadratic polynomials, Erg. Th. and Dynam. Syst. 12 (1992), 589-620. MR 1182664 (93h:58129)

School of Mathematical Sciences, Queen Mary, University of London, Mile End RoAd, London E1 4NS, United Kingdom

E-mail address: s.r.bullett@qmul.ac.uk

LatP/CMi, Université de Provence, 39 rue Frédéric Joliot-Curie, 13453 Marseille Cedex 13, France

E-mail address: phaissin@cmi.univ-mrs.fr 